ARTICLE

\title{
Tau activates microglia via the PQBP1-cGAS-STING pathway to promote brain inflammation
}

\author{
Meihua Jin (10) 1,5, Hiroki Shiwaku (10) 2,5, Hikari Tanaka (1) 1,5, Takayuki Obita (1) ${ }^{3}$, Sakurako Ohuchi (i) ${ }^{3}$, \\ Yuki Yoshioka (1) 1, Xiaocen Jin (1) 1, Kanoh Kondo (1) 1, Kyota Fujita (1) 1, Hidenori Homma (1) 1, \\ Kazuyuki Nakajima (i) ${ }^{4}$, Mineyuki Mizuguchi (i) ${ }^{3} \&$ Hitoshi Okazawa (i) ${ }^{1 凶}$
}

Brain inflammation generally accompanies and accelerates neurodegeneration. Here we report a microglial mechanism in which polyglutamine binding protein 1 (PQBP1) senses extrinsic tau $3 R / 4 R$ proteins by direct interaction and triggers an innate immune response by activating a cyclic GMP-AMP synthase (cGAS)-Stimulator of interferon genes (STING) pathway. Tamoxifen-inducible and microglia-specific depletion of PQBP1 in primary culture in vitro and mouse brain in vivo shows that $\mathrm{PQBP} 1$ is essential for sensing-tau to induce nuclear translocation of nuclear factor $\kappa B(\mathrm{NFKB}), \mathrm{NF \kappa B}$-dependent transcription of inflammation genes, brain inflammation in vivo, and eventually mouse cognitive impairment. Collectively, PQBP1 is an intracellular receptor in the cGAS-STING pathway not only for cDNA of human immunodeficiency virus (HIV) but also for the transmissible neurodegenerative disease protein tau. This study characterises a mechanism of brain inflammation that is common to virus infection and neurodegenerative disorders.

\footnotetext{
${ }^{1}$ Department of Neuropathology, Medical Research Institute and Center for Brain Integration Research, Tokyo Medical and Dental University, 1-5-45 Yushima, Bunkyo-ku, Tokyo 113-8510, Japan. ${ }^{2}$ Department of Psychiatry, Graduate School of Medical and Dental Sciences, Tokyo Medical and Dental University, 1-5-45 Yushima, Bunkyo-ku, Tokyo 113-8510, Japan. ${ }^{3}$ Faculty of Pharmaceutical Sciences, Graduate School of Innovative Life Science, University of Toyama, 2630 Sugitani, Toyama 930-0194, Japan. ${ }^{4}$ Department of Bioinformatics, Institute of Bioinformatics, Soka University, 1-236 Tangi-machi,

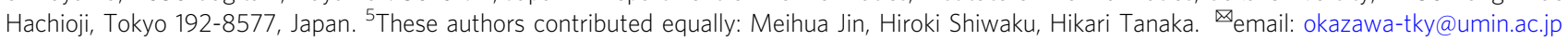


$\mathrm{N}$ eurodegenerative diseases are associated with inflammation of the brain ${ }^{1}$. Extracellular disease proteins like $A \beta^{2}$ and damage-associated molecular patterns (DAMPs) such as high mobility group box protein 1 (HMGB1) or $A \beta^{3,4}$ released from sick neurons are the first group of stimuli for activating Toll-like receptor 2/4 (TLR2/4) or Receptor For Advanced Glycation End-Products (RAGE) on the membrane of microglia ${ }^{5}$. Molecules presented on the plasma membrane of dying neurons such as phosphatidyl serine (PS), Growth Arrest Specific 6 (GAS6), and Protein S are the second group of stimuli for activating dendritic cells including microglia and macrophages that possess PS receptors and TAM (Tyrosine-Protein Kinase Receptor TYRO3/AXL Receptor Tyrosine Kinase/Tyrosine-Protein Kinase Receptor Mer) receptors ${ }^{6}$. Intercellular Adhesion Molecule-3 (ICAM3), located on the plasma membrane of T cells, is activated by dying neurons ${ }^{1}$ and is an indirect stimulus for dendritic cell-specific intercellular adhesion molecule-3-grabbing non-integrin (DC-SIGN) on microglia ${ }^{7}$. On the other hand, accumulating evidence indicate that inflammation accelerates neurodegenerative processes $^{1}$, suggesting a scheme of PCR-like amplification between degeneration and inflammation in the brains of neurodegenerative diseases.

Recent evidence from HIV infection sheds further light on the mechanism of microglia activation ${ }^{8}$. Reverse-transcribed single strand cDNA from the RNA genome of HIV interacts with PQBP1 as a sensor molecule, activates cGAS in their complex, and initiates transcription of inflammatory genes such as type-I interferons ${ }^{8}$. Subsequently, other proteins like Zinc Finger CCHC-Type Containing 3 (ZCCHC3) $^{9}$ and Ras-GTPaseActivating Protein SH3-Domain-Binding Protein 1 (G3BP1) ${ }^{10}$ were identified as sensors for double-strand genome DNA of herpes simplex virus type 1 and vaccinia virus, or for DNA inducing Aicardi-Goutières syndrome, respectively, and the cGAS-STING pathway was established as the third paradigm for activating microglia in general except in neurodegenerative diseases.

Interestingly, PQBP1 was originally identified as a binding protein to polyglutamine (polyQ) tract amino acid sequence that is expanded in a group of neurodegenerative diseases called polyQ diseases ${ }^{11-13}$. PQBP1 possesses a folded WW domain ${ }^{14-17}$ and an unfolded C-terminal domain categorized into intrinsically disordered proteins (IDPs) ${ }^{18-22}$. Loss of function of human PQBP1 by mutations causes intellectual disability and microcephaly ${ }^{23-26}$. PQBP1 functions as a regulator of RNA splicing, transcription, and DNA damage repair under physiological conditions ${ }^{13,27-30}$ which is related mainly to neuronal functions $s^{13,31-35}$, and possibly atherosclerosis ${ }^{36}$; however, it also functions as a sensor protein in macrophage and homologous cells of innate immune system for external molecules under certain pathological conditions ${ }^{8}$.

The accumulated evidence bridging the cGAS-STING pathway, PQBP1, and neurodegenerative proteins provide us with an idea that PQBP1, possessing characteristics of IDP, might sense (or interact with) disease proteins that have an unfolded and denatured structure as extrinsic proteins, to drive the cGAS-STING pathway in the innate immune system especially in microglia ${ }^{37}$. Recent findings about prion-like propagation of disease proteins in the brains seem to support this idea ${ }^{38-40}$

Here, we show that tau protein interacts with PQBP1 in vitro and drives innate immune response of primary microglia in a PQBP1-dependent manner and that PQBP1 expression in microglia is important for tau-induced inflammation in the brain by using a conditional knockout mouse model of PQBP1 in microglia. In addition to the sensing mechanisms by cell surface receptors for extracellular and intercellular proteins, these results show a third mechanism of inflammation in the brain of neurodegenerative diseases by an intracellular receptor, which could be generalized to multiple diseases since interaction rather than co-aggregation between the disease protein and PQBP1 occurs in Huntington's disease (HD) and spinocerebellar ataxia type $1(\mathrm{SCA} 1)^{12,13,41}$.

\section{Results}

Tau interacts with PQBP1 in microglia. Tau protein has various alternative-splicing forms (Supplementary Fig. 1), among which we selected human Tau 410 (a representative $3 \mathrm{R}$ tau) and Tau 441 (a representative $4 \mathrm{R}$ tau) for interaction with human PQBP1. Human and also mouse PQBP1 proteins possess a WW domain (WWD), polar amino acid-rich domain (PRD) including dinucleotide repeats and C-terminal domain (CTD) with intrinsically disordered protein regions (IDP/IDRs) (Fig. 1a). We selected human PQBP1 full-length form (PQBP1-FL), human PQBP1(1-94) including WWD, and human PQBP1 (94-176) including PRD and human PQBP1 (193-265) including CTD. Surface plasmon resonance (SPR) analysis revealed that Tau 410 and Tau 441 full-length proteins almost equally interacted with WWD of PQBP1 (Fig. 1b and Supplementary Table 1).

To identify the binding region between tau and PQBP1, we performed interaction analysis using NMR spectroscopy. We measured $2 \mathrm{D}^{1} \mathrm{H}_{-}{ }^{15} \mathrm{~N}$ HSQC NMR spectra of the full-length tau in the presence and absence of PQBP1. However, attempts to analyze the HSQC of full-length tau were complicated by extensive signal overlap and severe resonance line broadening Therefore, we divided Tau 410 into five fragments (Fig. 1a) and analyzed the interaction of these fragments with PQBP1(1-94) (Fig. 1c). The addition of PQBP1(1-94) resulted in chemical shift changes and resonance line broadening in the HSQC spectra of Tau(151-197), Tau(198-243), and the repeat region $\operatorname{Tau}(244-341)$ (Fig. 1c). In particular, Tau(151-197) and Tau(198-243), which are proline-rich regions, showed considerable resonance line broadening (Fig. 1c). This result is consistent with binding of the proline-rich sequence to the WW domain of PQBP1 in our previous study ${ }^{21}$. On the other hand, no significant changes were observed in the HSQC spectra of Tau(1-150) and Tau(342-410) (Fig. 1c).

Given that these results suggested that PQBP1 selectively bound to the proline-rich regions, we further made point mutations (P179A and P216A) at two possible consensus sequences $^{15,16}$ for interaction with WW domain of PQBP1 (Fig. 1d), and investigated their affinities to PQBP1 by SPR (Fig. 1b). The results revealed obvious decreases in the affinity of single mutants (Tau410 P179A or Tau410 P216A) and a more remarkable decrease in the affinity of double mutant (Tau410 P179A/P216A) (Fig. 1b).

In addition, the interaction between PQBP1 and Tau 410 was confirmed by qualitative and quantitative immunoprecipitation assays (Fig. 1e). Moreover, in vivo interaction between PQBP1 and Tau was confirmed by immunoprecipitation and immunohistochemistry using cerebral cortex tissue from a mouse model (R6/2) of Huntington's disease (Supplementary Fig. 2) that could be considered as a tauopathy ${ }^{42}$.

All of these results indicate that PQBP1 interacts with tau proteins by recognizing the proline-rich consensus sequences.

SPR analysis revealed that the interaction between PQBP1 and A $\beta 40$ was weaker than that between PQBP1 and Tau 410/441 (Supplementary Fig. 3). Interaction between PQBP1 and $\alpha$ synuclein was far weaker and not definitively determined (Supplementary Fig. 3).

Dynamics of Tau interaction with PQBP1 in microglia. Immunocytochemistry of primary mouse microglia revealed that 
a

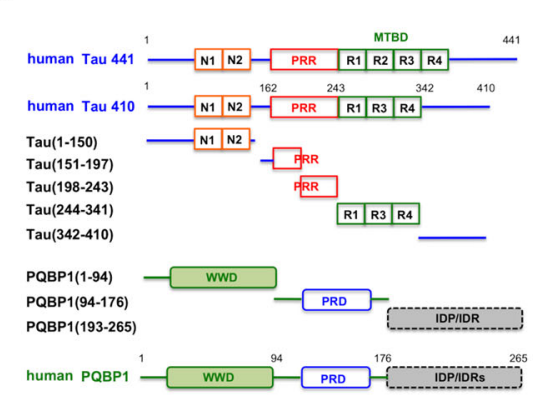

b
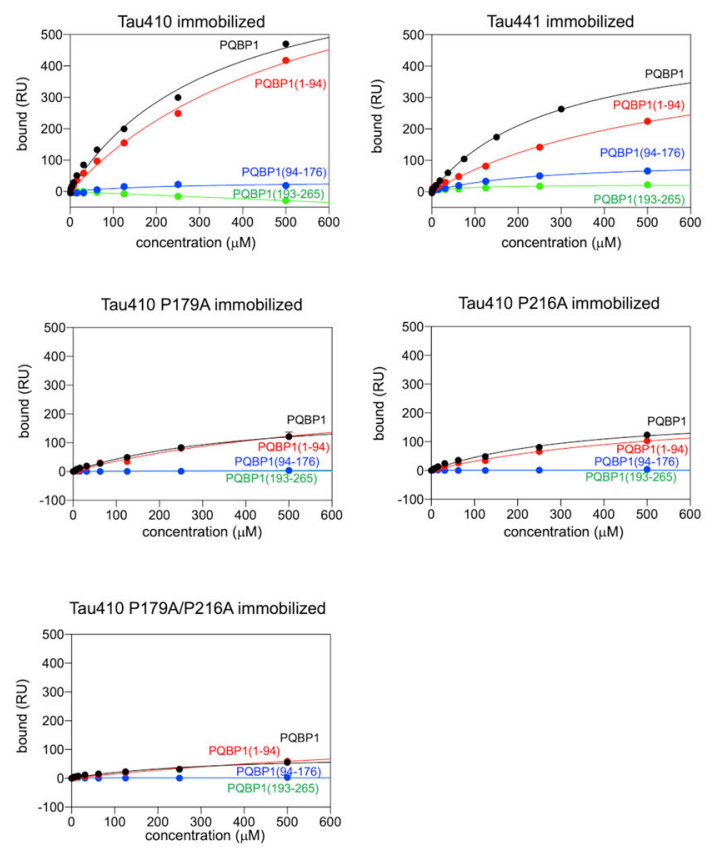

C

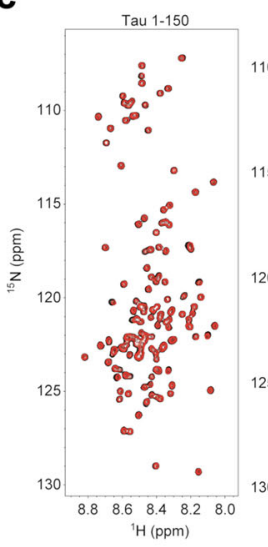

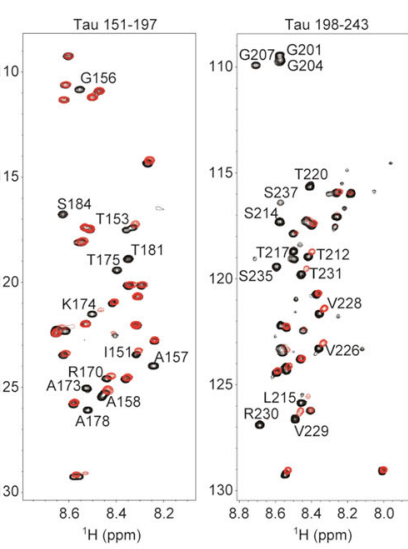
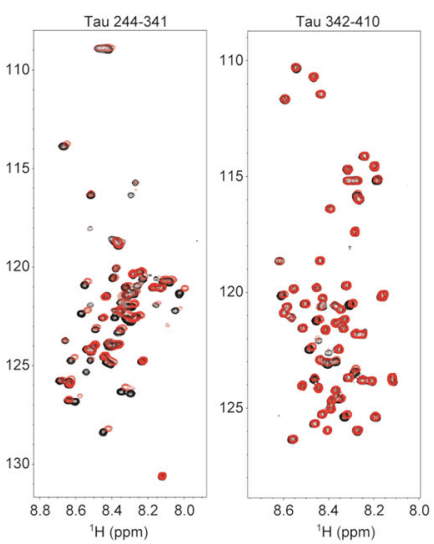

d

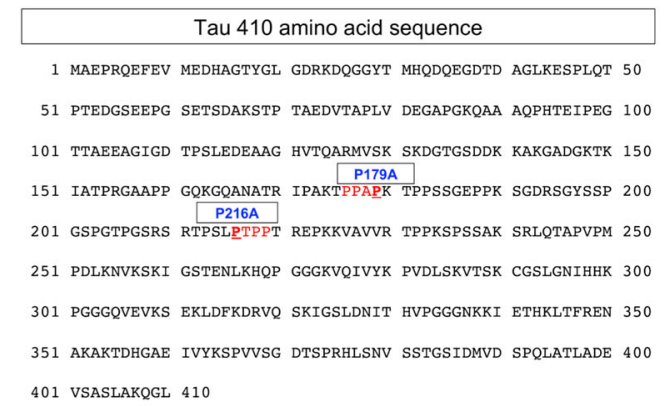

e
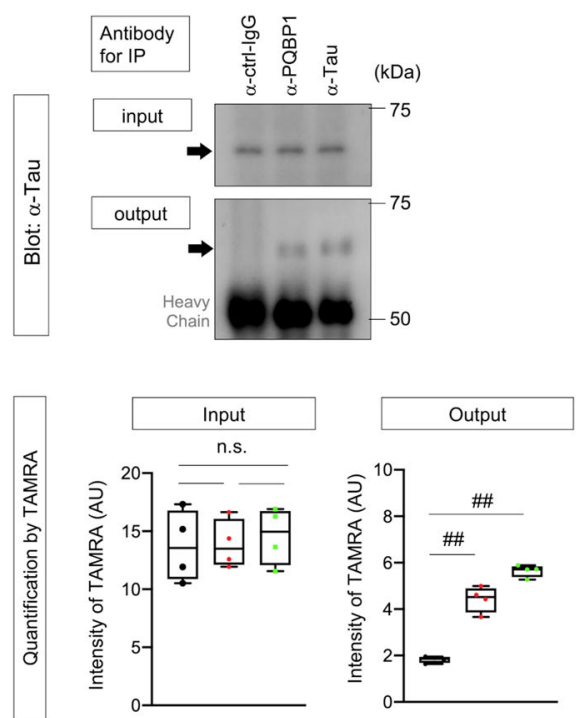

Antibody for IP

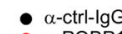

$\alpha-\mathrm{PQBP}$

\#\#: $P<0.01$

in Tukey's HSD test

incorporated four types of tau proteins from culture medium (Fig. 2c and Supplementary Movie 1).

First, PQBP1-EGFP was abundantly produced in rough ER surrounding the nucleus, and only a small portion was translocated into the nucleus (Fig. 2c and Supplementary Movie 1). At $0 \mathrm{~h}$ in the movie, Tau 410 or Tau 441 was added to the medium (Fig. 2c and Supplementary Movies 1 and 2, at $0 \mathrm{~h}$ ). Tau incorporation into microglia became obvious after $4 \mathrm{~h}$ (Fig. 2c). Interestingly, tau rapidly translocated to the rough ER, presumably via endosome-ER contact sites ${ }^{43-45}$. The signals of PQBP1-EGFP were pushed out to smooth ER that is located at the peripheral side of the rough ER (Fig. 2c and Supplementary Movie 2, at $4 \mathrm{~h}$ ), but then newly synthesized PQBP1-EGFP and tau were mixed up in rough ER (Fig. 2c and Supplementary 
Fig. 1 PQBP1 interacts with extrinsic Tau in microglia. a Schematic presentation of deletion mutants of tau and PQBP1 used for SPR and NMR analyses. N1/2 N-terminal domain, R1/2/3/4 C-terminal microtubule-binding domain, PRR proline-rich region, WWD WW domain, PRD polar amino acid-rich domain, IDP/IDR intrinsically disordered region. b Equilibrium binding curves of PQBP1 to Tau 410, Tau 441, Tau 410 P179A, Tau 410 P216A, and Tau 410 P179A/P216A monitored by SPR. Tau proteins were immobilized on sensor chip surfaces. The binding response at equilibrium was plotted against the concentration of PQBP1. Black, full-length PQBP1; red, PQBP1(1-94); blue, PQBP1 (94-176); green, PQBP1 (193-265). RU resonance unit. c ${ }^{1} \mathrm{H}-15 \mathrm{~N}$ HSQC spectra of Tau 1-150, 151-197, 198-243, 244-341, and 342-410 in the absence (black) and presence (red) of PQBP1(1-94). Amino acid residue numbers of tau are based on Tau 410. The molar ratio of Tau:PQBP1 (1-94) is 1:5. Residues showing significant chemical shift perturbation and line broadening of resonances are labeled in the HSQC spectra of Tau 151-197 and 198-243. Ctrl control, ppm parts per million. d Tau 410 amino acid sequence showing consensus motifs for interaction with PQBP1-WWD (red letter) and mutated prolines (underlined). e A representative western blot showing coprecipitation of tau with PQBP1 from a lysate of C57BL/6 microglia cultured for 3 days with $25 \mathrm{nM}$ non-aggregated Tau-TAMRA (upper panels). Quantitative immunoprecipitation was performed with C57BL/6 microglia cultured for 3 days with $25 \mathrm{nM}$ non-aggregated Tau-TAMRA. Input was the intensity of TAMRA-labeled Tau 410 in the C57BL/6 microglia lysate samples used for immunoprecipitation. Output was the intensity of TAMRA-labeled Tau 410 in samples after immunoprecipitation with anti-tau, anti-PQBP1, and human IgG. The fluorescence intensity of TAMRA-labeled Tau 410 was measured on a FLUOstar OPTIMA-6 microplate reader. n.s. not significant, AU arbitrary unit. $N=4$ per group. $P=0.9963$ (ctrl-lgG vs PQBP1), 0.8874

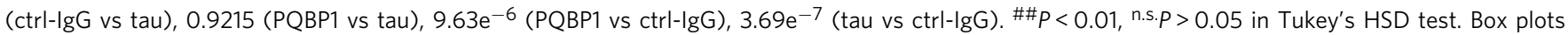
show the median, quartiles, and whiskers that represent data outside the 25 th to 75 th percentile range.

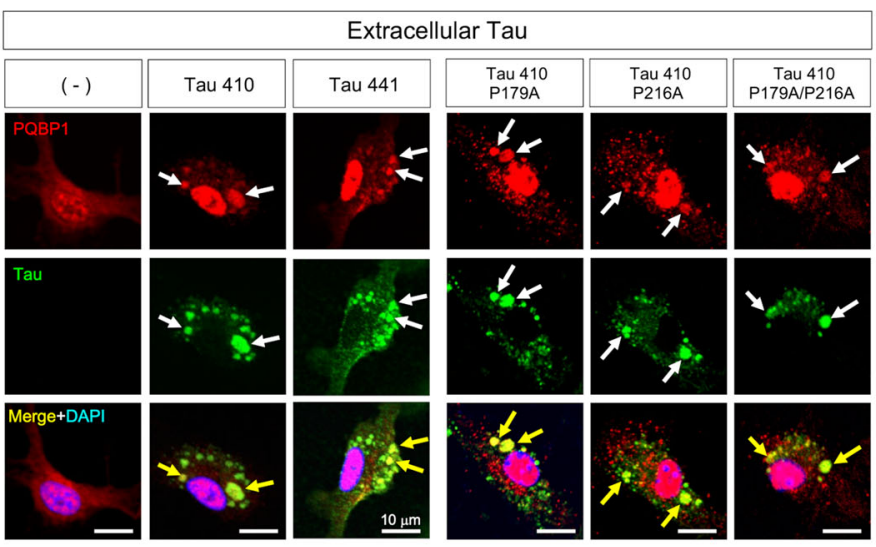

b

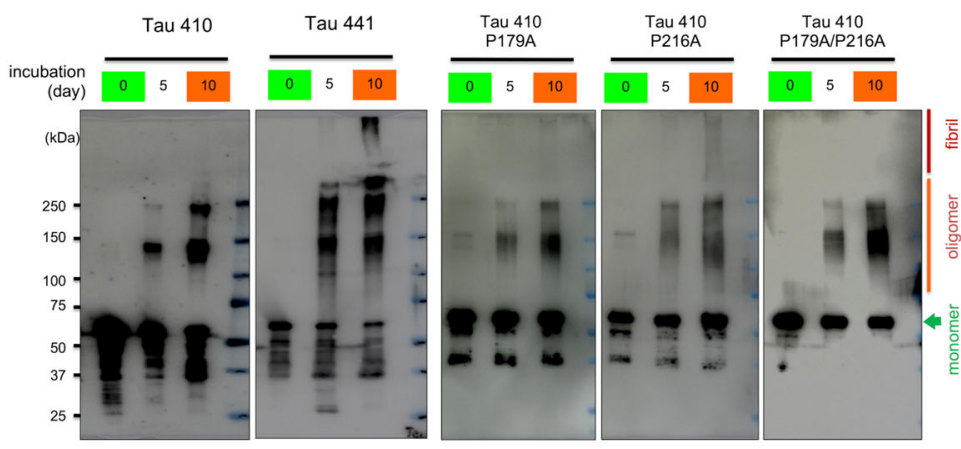

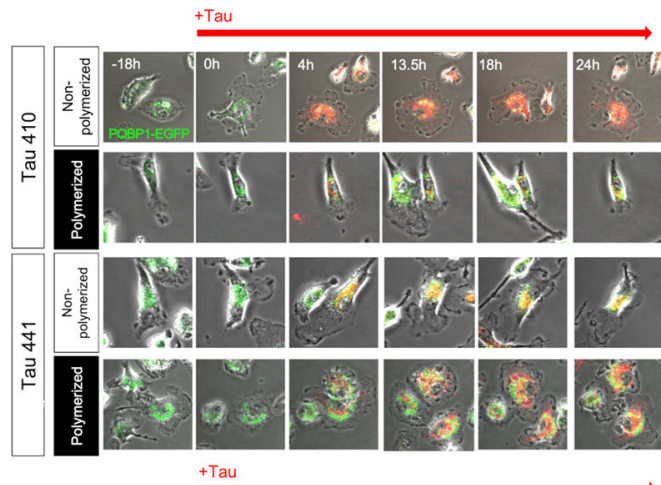

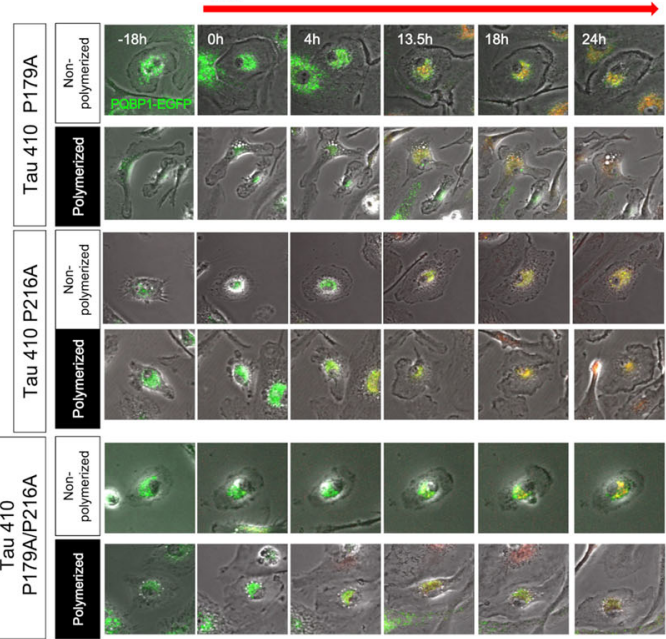

Fig. 2 Colocalization of PQBP1 and incorporated Tau in microglia. a Immunocytochemistry of primary microglia prepared from C57BL/6 mice cultured with Tau 410, Tau 441, Tau 410 P179A, Tau 410 P216A, or Tau 410 P179A/P216A proteins in the medium. Colocalization of PQBP1 and extrinsic tau was detected frequently. The experiments were repeated four times independently with similar results. $\mathbf{b}$ In vitro polymerization of Tau 410, Tau 441, Tau 410 P179A, Tau 410 P216A, and Tau 410 P179A/P216A full-length proteins. Only Tau 441 and Tau 410 P216A partially formed fibrils after 10 days of incubation, while the other full-length protein did not. The experiments were repeated four times independently with similar results. c Images of normal microglia before and after addition of polymerized or non-polymerized Tau 410, Tau 441, Tau 410 P179A, Tau 410 P216A, or Tau 410 P179A/P216A, among which three species are shown in Supplementary Movies 1-3. h hour.

Movie 2, after $12 \mathrm{~h}$ ). Thereafter, the signals of PQBP1-Tau complex diffusely leaked from ER to the cytosol (Fig. 2c and Supplementary Movie 2, after $13 \mathrm{~h}$ ).

Basically, Tau 410 and Tau 441 behaved similarly (Fig. 2c and Supplementary Movie 1). However, tau and PQBP1 signals merged immediately in ER in the case of Tau 410 monomer, while the merge of signals was delayed in the case of polymerized
Tau 441 (Fig. 2c and Supplementary Movies 1 and 2). Interestingly, STING is located in ER (subcellular location in UniProt https://www.uniprot.org/uniprot/Q86WV6) while cGAS is located in cytosol (subcellular location in UniProt https:// www.uniprot.org/uniprot/Q8N884) after stimulation. Therefore, the Tau-PQBP1-cGAS-STING complex could be formed in the prograde order of the dynamics of tau observed above (Fig. $2 \mathrm{c}$ 
and Supplementary Movie 1), or the Tau-PQBP1-cGAS-STING complex might be formed in retrograde order ${ }^{8}$ during the reflux from cytosol to ER that may occur under the collapse of the ER-cytosol border (Fig. 2c and Supplementary Movie 2).

In the case of binding mutants of tau (Tau410 P179A, Tau410 P216A, Tau410 P179A/P216A) in monomer and polymerized states, live imaging analysis of normal primary microglia suggested a tendency for delayed merging between PQBP1 and the binding mutants of Tau in ER (Fig. $2 \mathrm{c}$ and Supplementary Movie 3).

It is very interesting that direct incorporation of extrinsic tau to rough ER is quite homologous to the direct incorporation of coronavirus RNAs causing COVID-19, MERS, and SARS into rough ER for the formation of double membrane vesicle $(\mathrm{DMV})^{46,47}$.

Tau activates cGAS-STING pathway in microglia. To examine whether extrinsic tau proteins activate cGAS-STING pathway in microglia, we first confirmed nuclear translocation of nuclear factor $\kappa \mathrm{B}(\mathrm{NF} \kappa \mathrm{B})$ (Fig. 3a) at the final step of the cGAS-STING pathway ${ }^{8,48}$. As expected, we observed nuclear translocation of NFkB-p65 in more than $80 \%$ of microglia by the addition of Tau 410/441 proteins to the culture medium (Fig. 3a). Meanwhile, addition of binding mutants of Tau did not induce nuclear translocation of NFkB so efficiently (Fig. 3a). Moreover, polymerized tau proteins, after incubation for 10 days, induced nuclear translocation of $\mathrm{NF \kappa B}$ less efficiently than the monomer state of tau (no polymerization) (Fig. 3a). We calculated the ratio of nuclear NFkB-positive microglia in all cases (Fig. 3b).

A similar difference was observed in the induction of $\operatorname{Tnf}$ and Isg54 gene expression in microglia (Fig. 3c). Tau 410 and Tau 441 isoforms did not show obvious differences in inducing nuclear translocation of NFKB and Isg54 gene expression (Fig. 3b, c), while Tau 441 monomer and polymer did not efficiently induce Tnf gene expression (Fig. 3c). The binding mutants of tau did not efficiently induce $\operatorname{Tnf}$ and Isg54 gene expression in microglia (Fig. 3c).

The independency of human Tau 410 and Tau 441 isoforms in activating the cGAS-STING pathway in mouse microglia could be a common mechanism across various tauopathies including Alzheimer's disease (AD), given that different composition of $4 \mathrm{R} /$ $3 \mathrm{R}$ tau isoforms recapitulate progression patterns of the corresponding human diseases independently of tau species and mouse strains ${ }^{49}$. Tau-induced activation of cGAS-STING pathway might underlie a more general inflammatory response that is independent of progression patterns of tauopathy in the central nervous system.

PQBP1 is essential for tau-induced cGAS-STING activation in microglia. Combination of these two lines of results prompted us to test further whether PQBP1 is essential for tau-induced cGAS-STING activation. We generated microglia-specific and tamoxifen-inducible PQBP1 conditional knockout mouse

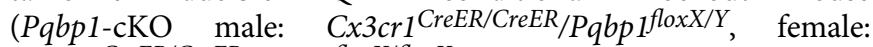
Cx3cr $1^{C r e E R / C r e E R} / P q b p 1^{\text {floxX/floxX }}$ ) by crossing Pqbp1-floxed mice (Supplementary Fig. 4a) and tamoxifen-inducible Cx3cr ${ }^{C r e E R}$ mice (Supplementary Fig. 4b), in which tamoxifen-inducible depletion of PQBP1 in microglia was confirmed by immunohistochemistry of cerebral cortex tissues (Supplementary Figs. 5 and 6).

Primary microglia prepared from newborn homozygous Pqbp1-cKO mice were stimulated by addition of Tau 410/441 in the culture medium (Fig. 4a). While Tau 410 and Tau 441 induced nuclear translocation of NFKB in all cells of microglia prepared from Pqbp1-cKO mice in the absence of tamoxifen, tau proteins induced nuclear translocation of $\mathrm{NFkB}$ only in one half of microglia in the presence of tamoxifen (Fig. 4b). The inhibitory effect on nuclear translocation of NFKB was similarly observed in microglia transfected with siRNA of cGAS or STING (Fig. 4b). Quantification of the number of cells with nuclear translocation of NFkB (p65) supported this conclusion (Fig. 4c). Western blot revealed an increase in the active form of NFKB (pSer536-NFkBp65) by Tau 410/441 in normal microglia (Fig. 4d). Moreover, western blot analysis with an antibody against the active form of IRF3 (pSer396-IRF3) revealed that IRF3 activation, another downstream transcription factor of the cGAS-STING pathway ${ }^{50}$ (Fig. 4d). However, such activation of NFkB and IRF3 were suppressed by PQBP1 depletion, knockdown of cGAS or knockdown of STING in microglia (Fig. 4d).

It is noteworthy that depletion of PQBP1 and suppression of cGAS or STING reduced nuclear translocation of NFkB to nearly $50 \%$ but not completely, suggesting the existence of pathway(s) other than the PQBP1-cGAS-STING pathway that could induce $\mathrm{NF \kappa B}$ activation.

Other functions of PQBP1-depleted microglia, such as chemotaxis (Fig. 4e) and phagocytosis (Fig. 4f), were unchanged, while it remains possible that other functions of microglia may be affected by depletion of PQBP1, given that PQBP1 is involved in transcriptional $12-14$ and post-transcriptional gene expression $^{26-30,34,35}$

PQBP1 is essential for cGAS to recognize Tau and for microglia activation. Intracellular dynamics and localization of multiple cGAS-STING complex components have not been elucidated in detail. However, physical colocalization of tau and cGAS is essential for recognition of target proteins coming from the extracellular space. We found in wild-type primary microglia that Tau 410/441 monomer proteins taken up from the culture medium, colocalized with cGAS after AT8 antibody-recognizable phosphorylation (Fig. 5a). Meanwhile, in Pqbp1-cKO microglia, cGAS protein was not recruited to the foci of phosphorylated tau proteins (Fig. 5a). The tendency was confirmed by quantification of the ratio of Tau-cGAS colocalized microglia to all microglia (Fig. 5b). The result indicated that PQBP1 guides cGAS proteins to tau protein foci in microglia.

Next, we tested the effect of PQBP1 on transcriptional function of NFkB under the stimulation of Tau 410/441 by performing a luciferase assay for the activity of $\mathrm{NFKB}$ cis-element and quantitative reverse transcription polymerase chain reaction (RT-qPCR) for expression of target genes of NFkB (Fig. 5c). Luciferase assay using the reporter plasmid containing the consensus cis-element for NFkB was activated by Tau 410/ 441 stimulation, and revealed that partial deficiency of PQBP1, cGAS, and STING suppressed the activation of NFKB cis-element (Fig. 5d). Internal control (renilla) was used for correcting the specific signal of firefly in the luciferase assays (Fig. 5d). Pqbp1cKO microglia and siRNA-transfected microglia were used to prove the essential function of PQBP1, cGAS, and STING for activation of NFkB cis-element (Fig. 5d). RT-qPCR also revealed that Tau 410/441 stimulation transcriptionally upregulated target genes of NFkB, such as Tnf, Isg54, Cxcl10, and Ifn $\beta$, indicating that PQBP1, cGAS, and STING were essential for the upregulation (Fig. 5e).

PQBP1 depletion in microglia rescues tau-induced brain inflammation. Finally, we examined whether PQBP1 intracellular receptor senses extrinsic tau and activates the cGAS-STING pathway in mouse brains in vivo. For this purpose, we prepared tamoxifen-inducible microglia-specific homozygous Pqbp1-cKO mice (Supplementary Fig. 4), in which PQBP1 is completely 
a

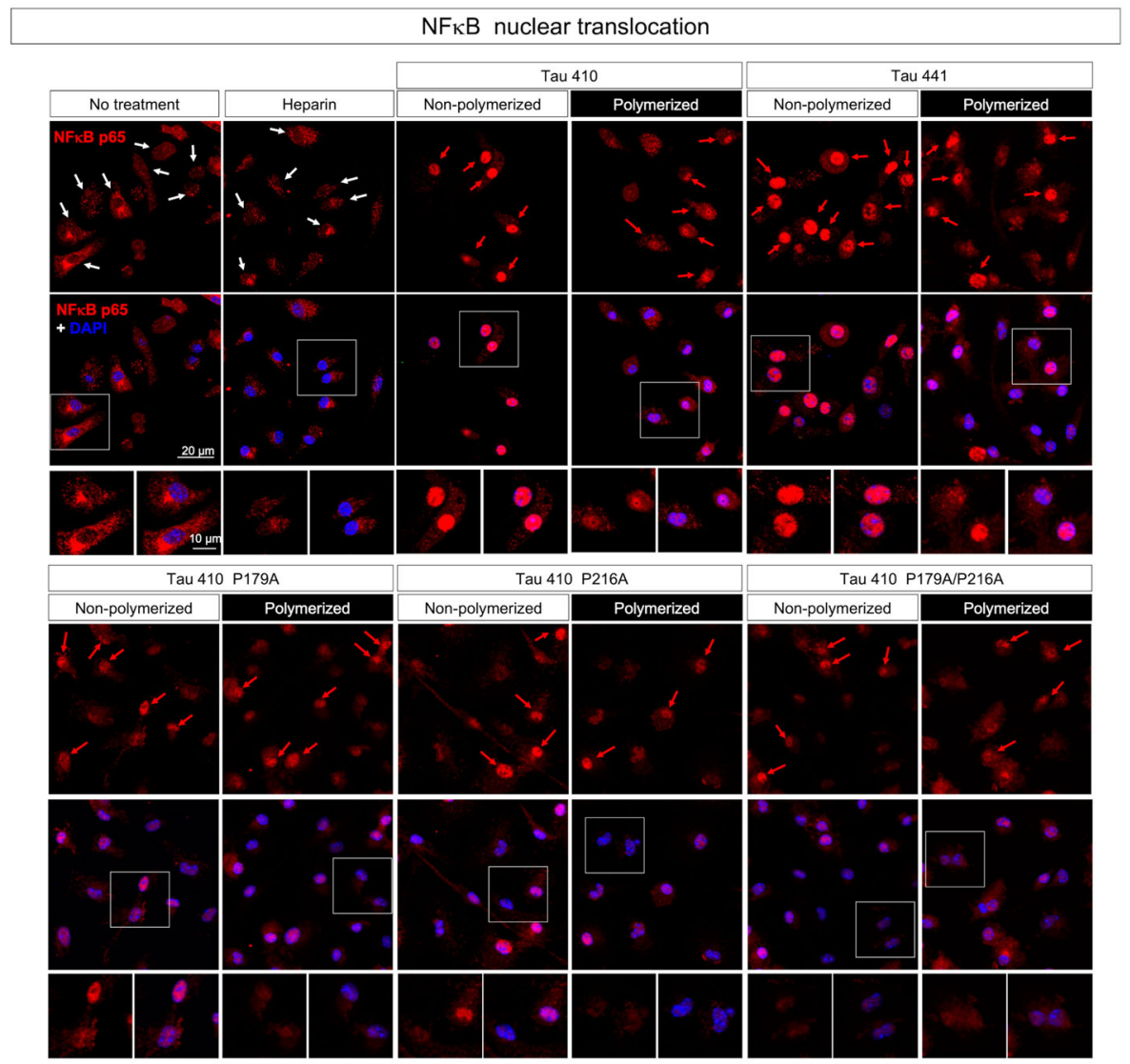

b

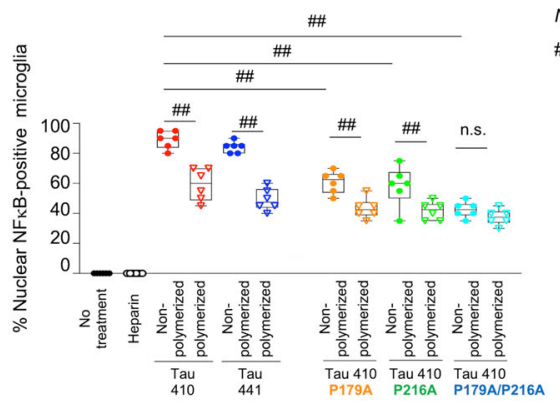

$N=5, n=20$ (cells)

\#\#: $P<0.01$ in Tukey's HSD test

C

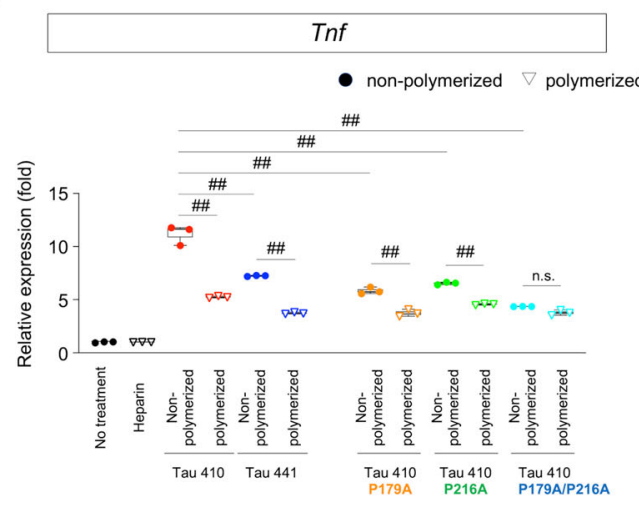

depleted in microglia (Supplementary Figs. 5 and 6). Tamoxifen by itself was not so toxic to influence the number of microglia at the dose used to induce Cre (Supplementary Fig. 6, Iba1 staining).

We prepared four groups of mice: $P q b p 1-c K O$ injected with Tau 410 monomer after tamoxifen treatment, Pqbp1-cKO injected with phosphate-buffered saline (PBS) after tamoxifen treatment, $P q b p 1-c K O$ injected with Tau 410 monomer after

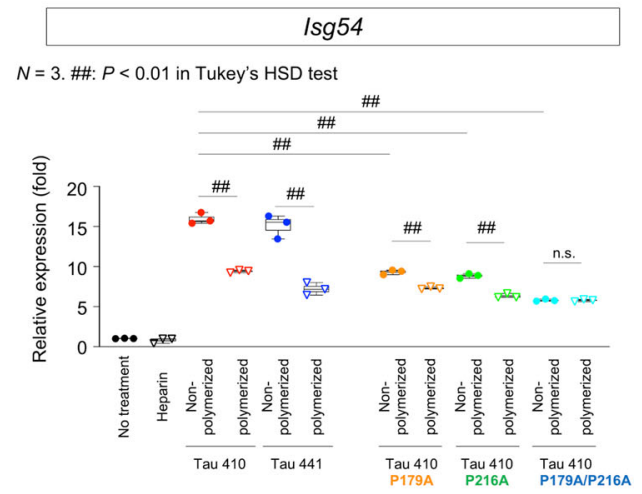

mock treatment, and Pqbp1-cKO injected with PBS after mock treatment. We injected Tau 410 monomer or a similar volume of solvent to bilateral entorhinal cortex $(2.2 \mu \mathrm{g} / \mu \mathrm{l}, 1 \mu \mathrm{l} /$ injection, $4.4 \mu \mathrm{g} /$ mouse) (Fig. 6a).

Immunohistochemistry of control group brains (non-induced Pqbp1-cKO injected with Tau 410 monomer) revealed tau incorporation, Tau-PQBP1 colocalization, nuclear translocation 
Fig. 3 Extrinsic Tau proteins induce nuclear translocation of NFKB and expression of pro-inflammatory genes in microglia. a Immunocytochemistry of primary microglia prepared from newborn C57BL/6 mice reveals nuclear translocation of NFkB at $48 \mathrm{~h}$ after addition of recombinant full-length Tau 410 , Tau 441, Tau 410 P179A, Tau 410 P216A, or Tau 410 P179A/P216A protein in the non-polymerized or polymerized state to culture medium. Images were obtained by confocal microscopy. Red or white arrows indicate microglia with or without nuclear translocation of NFkB. Lower panels show high magnification of representative cells. In the presence of tau proteins in culture medium, nuclear translocation of NFkB was induced in most microglia, while in the absence of tau proteins NFKB remained in the cytoplasm. $\mathbf{b}$ The effects of various tau species in the polymerized and non-polymerized state on nuclear translocation of NFKB were compared. Aggregation of Tau 410/441 reduces the frequency of NFKB nuclear translocation. PQBP1-binding proline single mutants (Tau 410 P179A, Tau 410 P216A) partially, while double proline mutant (Tau 410 P179A/P216A) substantially, lost the ability to induce NFKB nuclear translocation. $N=5, n=20$ (cells). $\# P<0.01$ in Tukey's HSD test. c Isoforms, mutations, and aggregation states of tau affect induction of Tnf and Isg54 gene expression. Consistent with immunocytochemistry of NFkB, non-polymerized Tau 410 induced pro-inflammatory genes most efficiently, while Tau 441 possesses $60-80 \%$ of the induction ability. PQBP1-binding proline single mutants (Tau 410 P179A, Tau 410 P216A) partially, while double proline mutant (Tau 410 P179A/P216A) substantially, lost transactivation of Tnf and Isg54 gene expression. RT-qPCR reverse transcriptase quantitative polymerase chain reaction. $N=3$. ${ }^{\#} P<0.01$ in Tukey's HSD test. Box plots show the median, quartiles, and whiskers that represent data outside the 25th to 75 th percentile range.

of NFkB (p65), and cGAS recruitment to the complex were observed in microglia gathering to injected area of entorhinal cortex (Fig. 6b, left panels). On the other hand, PQBP1 colocalization to tau, nuclear translocation of $\mathrm{NF \kappa B}$ or cGAS recruitment to the complex was not found in microglia of tamoxifen-induced Pqbp1-cKO mice (Fig. 6b, middle panels). Quantification of such changes in microglia confirmed these conclusions (Fig. 6b, right graphs). Microglia activation with nuclear translocation of NFKB occurred at a high frequency (89\%) when tau was incorporated (Fig. $6 \mathrm{~b}$, right graphs), while the ratio was low (6.8\%) among total microglia (Tau-positive and Taunegative microglia) (Supplementary Fig. 7) because tau incorporation was not so efficient.

The similarity to the disease-specific microglia ${ }^{51}$ to microglia activated in tau was also checked by using anti-lipoprotein lipase (LPL) antibody (Supplementary Fig. 8). We observed LPLpositive microglia similar to the disease-specific microglia reported previously ${ }^{51}$, and when tau was injected into mouse brains, their total number decreased in the absence of PQBP1 according to total number of microglia (Supplementary Fig. 8), while the ratio of LPL-positive microglia among total microglia was unchanged (Supplementary Fig. 8). LPL in microglia is essential for its lipid uptake and regulates immune reactivity of ordinary microglia $^{52}$. LPL immunoreactivity is linked to amyloid plaques while it is not associated with neurofibrillary tangles ${ }^{53}$. These notions might explain why tau injection did not induce a disproportionate increase of LPL-positive microglia.

RT-qPCR of Tnf and Isg54 with entorhinal cortex reconfirmed enhanced brain inflammation in the control mouse group (Pqbp1-cKO after mock treatment injected with Tau 410 monomer) while it was recovered by microglia-specific depletion of PQBP1 (Fig. 6c). Y-maze test and Morris water maze test supported that microglia-specific depletion of PQBP1 recovered cognitive symptoms based on the extracellular tau-induced brain inflammation (Fig. 6d).

In vivo live imaging of microglia by two-photon microscopy with $\mathrm{B} 6.129 \mathrm{P} 2(\mathrm{Cg})-C \times 3 \mathrm{cr} 1^{\text {tm1Litt }} / \mathrm{J}\left(C X_{3} C R-1^{G F P}\right)$ mice in which microglia were labeled by endogenous $C x 3 c r 1$ enhancer/promoter-driven EGFP protein revealed that microglia incorporated red particles of TAMRA-NHS-labeled Tau 410 full-length protein injected in monomer state (Fig. 6e). A part of such microglia (yellow cell body) with Tau 410 signals became active in movement of branches and enlarged into cell bodies (Fig. 6e, upper panels). Such activation of microglia by tau protein was suppressed by co-injection of siRNA against PQBP1 (Fig. 6e, lower panels) that efficiently suppressed PQBP1 (Fig. 6f).

PQBP1-binding mutations of Tau reduce inflammatory responses of microglia in brain. Inflammatory responses of microglia to tau proteins were also reduced in vivo when they were mutated at the binding motifs to PQBP1 (Fig. 7), similar to the case in vitro (Fig. 3). We injected normal Tau 410 or the binding mutants of tau (Tau410 P179A, Tau410 P216A, or Tau410 P179A/P216A) to bilateral entorhinal cortex of noninduced or tamoxifen-induced microglia-specific homozygous Pqbp1-cKO mice (Fig. 7a). Colocalization of tau proteins to PQBP1 (Fig. 7b) was abolished in tamoxifen-induced Pqbp1-cKO mice (Fig. 7c). Recruitment of cGAS was reduced to PQBP1-Taudouble-positive foci, when tau binding mutants were injected (Fig. 7b), while the recruitment of cGAS was completely abolished in tamoxifen-induced Pqbp1-cKO mice (Fig. 7c). The final outcome, nuclear translocation of $\mathrm{NFKB}$, was decreased in the case of tau-binding mutants (Fig. 7b), whereas it was not completely abolished in tamoxifen-induced Pqbp1-cKO mice (Fig. 7c), suggesting the existence of another pathway inducing NFkB activation, in addition to the pathway based on the tau-PQBP1-cGAS complex.

Quantitative RT-PCR of Tnf and Isg54 with brain tissues of Tau-injected area reconfirmed the reduced inflammatory responses of microglia to tau-binding mutants (Fig. $7 \mathrm{~d}$ ). The residual inductions of Tnf and Isg54 by Tau410 P179A or Tau410 P216A were further suppressed by depletion of PQBP1 in tamoxifen-induced microglia-specific Pqbp1-cKO mice (Fig. 7e).

Relationship between PQBP1 and LRP1 or TREM2. We addressed the mechanistic relationship of PQBP1 to the molecules playing critical roles in Tau-mediated pathology, lowdensity lipoprotein receptor-related protein 1 (LRP1) and triggering receptor expressed on myeloid cells 2 (TREM2). LRP1 is located at Clathrin-coated pit of cell membranes, and incorporates relatively large extracellular molecules, including lipoproteins, proteinase and inhibitor complexes, matrix proteins and other proteins into the cell via endosomes, and functions in macrophage for atherosclerosis ${ }^{54}$. LRP1 is also expressed in microglia ${ }^{55}$ and regulates the microglial immune response $e^{56,57}$. LRP1 was recently demonstrated as a dominant tau receptor ${ }^{58}$. TREM2 is a membrane receptor coupled with DNAX-activating protein $(12 \mathrm{kDa}$; DAP12) that transduces various signals including ERK and PI3K that activate $\mathrm{NF \kappa B}^{59,60}$. It has been identified as a risk factor for $\mathrm{AD}^{61,62}$, and is expressed in microglia to impede tau seeding and tau pathology in mouse models ${ }^{63-65}$.

We transfected the double nickase plasmid that expressed green fluorescent protein (GFP) for identification of transfected cells (Fig. 8a, left images) and almost completely knocked out Lrp1 or Trem 2 gene (Fig. 8a, right graphs) in microglia prepared from tamoxifen-inducible microglia-specific Pqbp1-cKO mice. Control plasmid expressed scrambled 20 nt gRNA. NFkB activity was monitored by two reporter plasmids expressing red 
a

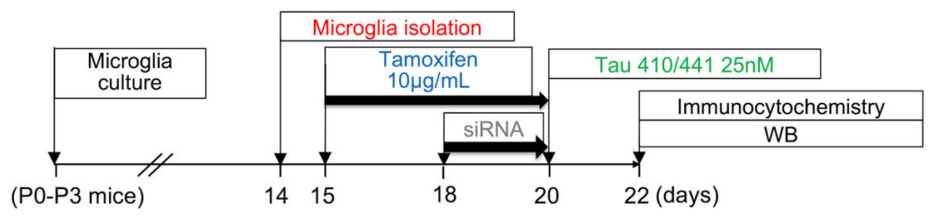

b

Tau 410
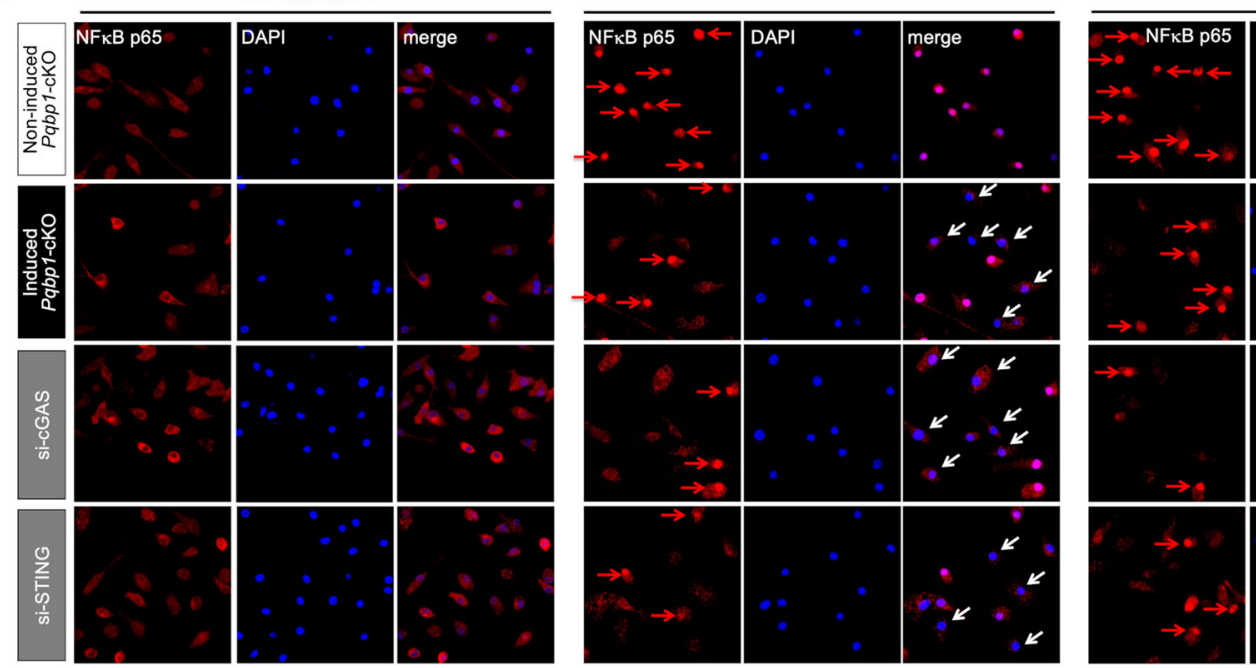

Tau 441

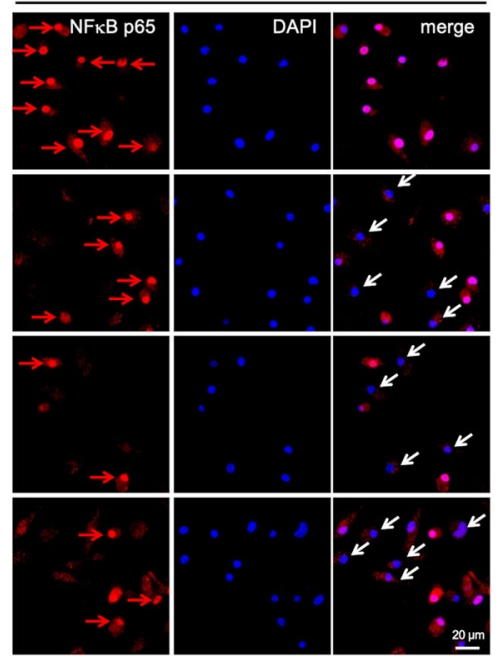

C

$$
\begin{aligned}
& \text { Non-induced Pqbp 1-cKO si-cGAS } \\
& \text { Induced Pqbp1-cKO } \\
& N=6 \text { (visual fields), \#\# } P<0.01 \text { in Tukey's HSD test } \\
& \text { (vs non-induced Pqbp1-cKO) }
\end{aligned}
$$

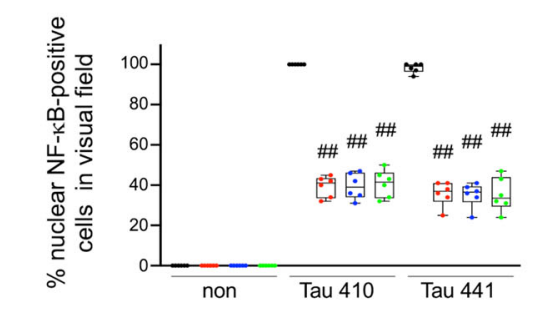

d

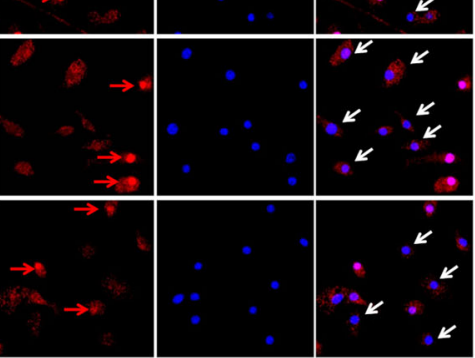

e
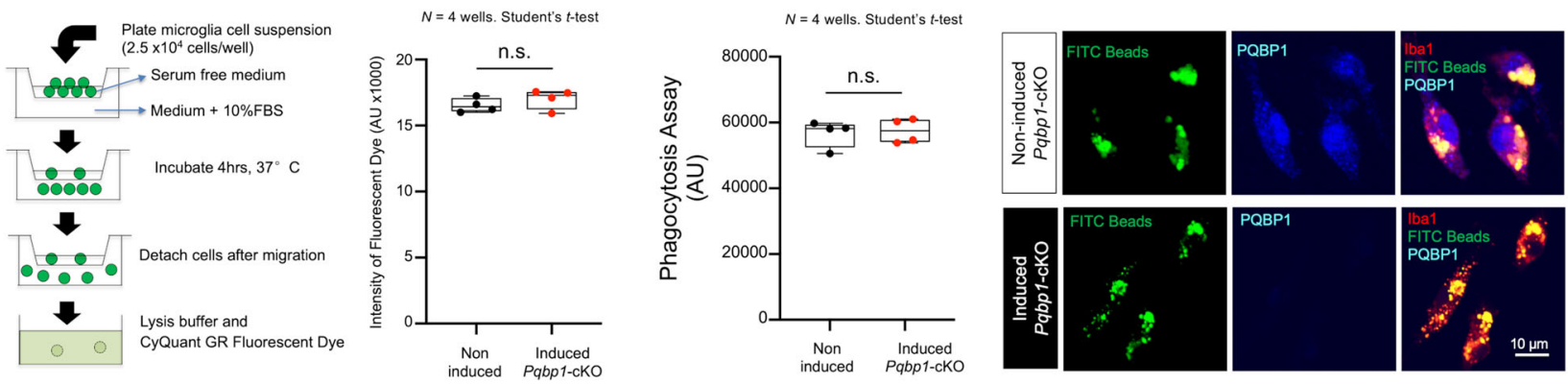

Fig. 4 PQBP1-cGAS-STING pathway mediates Tau-induced NFKB activation in microglia. a Protocol of tamoxifen-induced Pqbp1 knockout in primary microglia prepared from newborn Pqbp1-cKO mice. WB western blot. b Effect of PQBP1 deficiency, cGAS knockdown, and STING knockdown on Tau 410/ 441-induced nuclear translocation of NFKB in microglia. Activated microglia with nuclear NFkB are indicated with red arrows. Non-activated microglia are indicated with white arrows. Images were obtained by confocal microscopy. c Quantitative analyses of \% nuclear NFkB-positive microglia in six visual fields in normal condition, PQBP1 deficiency, cGAS knockdown, and STING knockdown. $N=6$ (visual fields). \#\# $P<0.01$ in Tukey's HSD test (vs non-induced Pqbp1-cKO). d Western blots reveal activation of NFkB (pSer536-p65) and IRF3 (pSer396-IRF3) after culture with non-polymerized Tau 410/441 in normal microglia, but not in tamoxifen-induced Pqbp1-cKO, siRNA-mediated cGAS-KD, or siRNA-mediated STING-KD microglia. This experiment was repeated independently, three times, with similar results. e Effect of PQBP1 depletion on microglia migration. FBS fetal bovine serum. $N=4$ wells. Statistical test: Student's t-test (two-sided). f Effect of PQBP1 depletion on microglia phagocytosis by using FITC-beads. AU arbitrary unit. $N=4$ wells. For statistical analysis, a Student's t-test (two-sided) was performed. Box plots show the median, quartiles, and whiskers that represent data outside the 25th to 75th percentile range. 
a

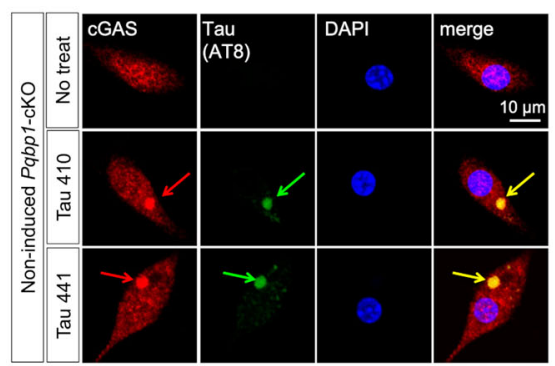

C
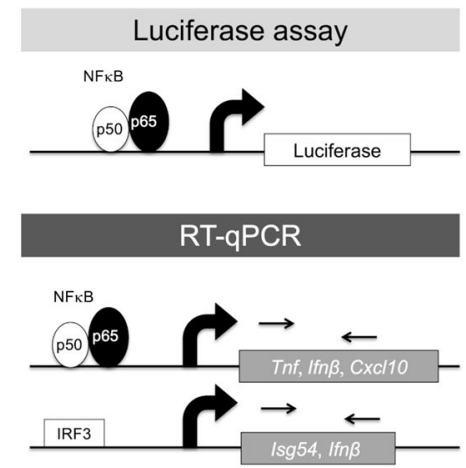

e
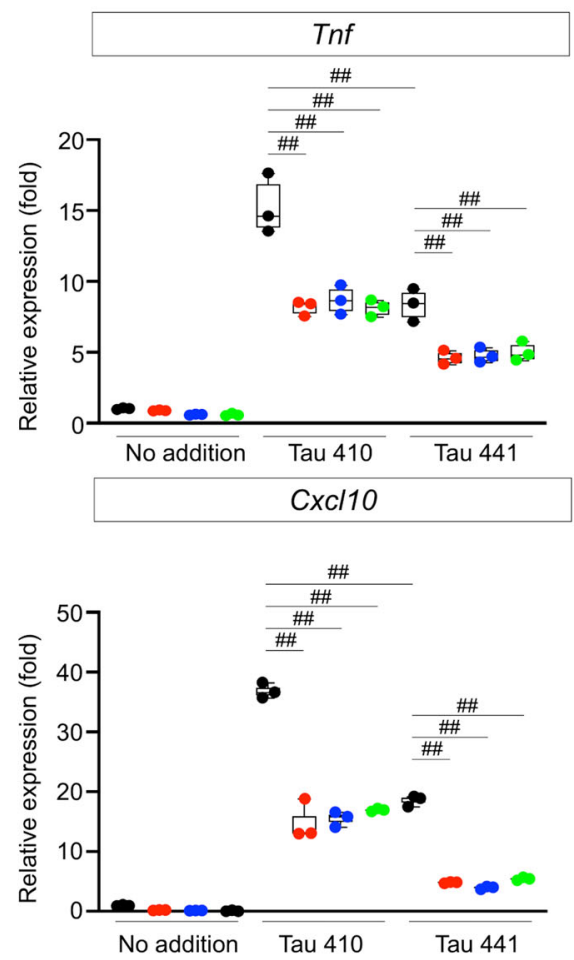
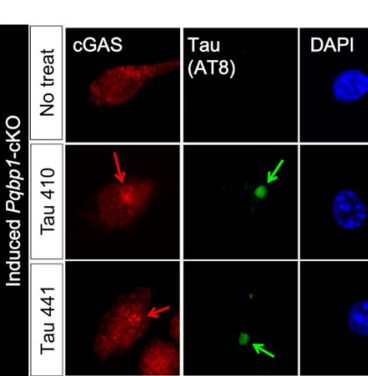

merge

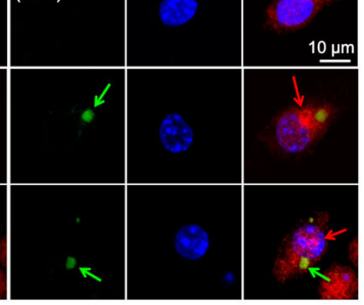

d
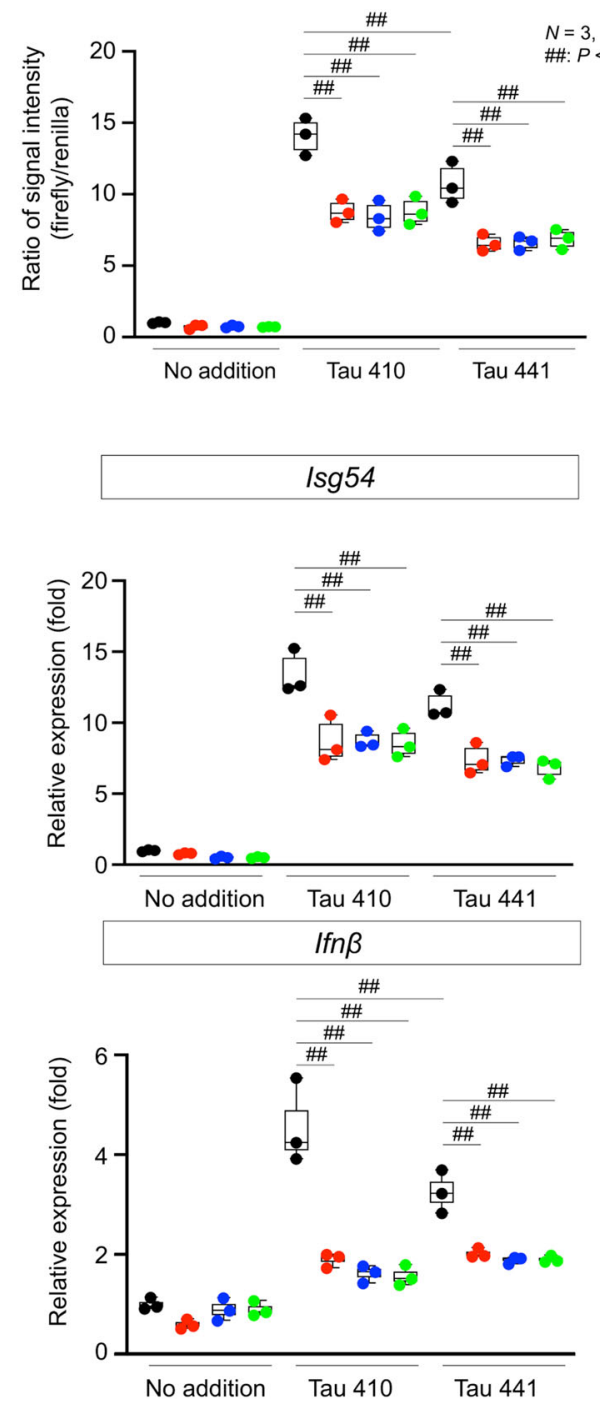

fluorescent protein (RFP) or luciferase driven by NFKB promoter (Fig. 8b). NFkB activation in microglia by addition of Tau 410 to the culture medium was suppressed by $\operatorname{Lrp1} 1-\mathrm{KO}$ or Trem $2-\mathrm{KO}$ in the RFP signal assay, as expected (Fig. 8c). In the case of additional Pqbp1-cKO by tamoxifen, NFkB activity was unchanged in Lrp1-KO microglia, but further suppressed in Trem2-KO (Fig. 8c). The discrepancy between NFkB activities in Lrp1-KO and Trem2-KO cells under the additional Pqbp1-cKO was also confirmed by luciferase assay (Fig. 8d). These genetic analyses indicated that PQBP1 and LRP1 are in the same pathway, while PQBP1 is independent of TREM2 (Fig. 8e).

Direct and indirect effects of Tau on neuronal death. MAP2 immunohistochemistry of Tau 410-injected mice revealed that non-microglia cells incorporating tau (Fig. 6b, middle panels) were mostly neurons (Fig. 9a). This finding, together with accumulated knowledge of tau propagation ${ }^{66}$, prompted us to comprehensively address relationship of the direct effect of tau incorporated into neurons and the indirect effect of tau-induced 
Fig. 5 PQBP1 is essential for recruiting cGAS to Tau. Confocal microscopy images of primary Pqbp1-cKO microglia stained with anti-cGAS and -tau antibodies. a Cytoplasmic tau foci after incorporation recruit cGAS in the presence of PQBP1 (left panels), while in the absence of PQBP1, cGAS is mislocalized and not merged with cytoplasmic foci of tau, which is phosphorylated after endocytosis. $\mathbf{b}$ Quantification of cGAS-tau colocalized cells in four wells of non-induced and tamoxifen-induced Pqbp1-cKO microglia after tau addition to primary culture medium. $N=4$. \#\#P<0.01 in Tukey's HSD test. c Schemes showing the concepts of the luciferase assay used to monitor NFKB activity under tau stimulation and PQBP1-cGAS-STING pathway perturbation (d) and of RT-qPCR used to monitor expression levels of NFkB-target genes (e). d Luciferase assay to monitor NFkB activity under Tau 410/ 441 stimulation together with PQBP1-cGAS-STING pathway perturbation by tamoxifen-induced Pqbp1-cKO, siRNA against cGAS and siRNA against STING.

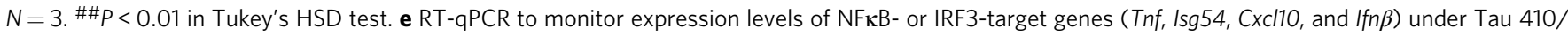
441 stimulation together with PQBP1-cGAS-STING pathway perturbation by tamoxifen-induced Pqbp1-cKO, siRNA against cGAS, and siRNA against STING. $N=3$. \#\#P<0.01 in Tukey's HSD test. Box plots show the median, quartiles, and whiskers that represent data outside the 25th to 75th percentile range.

microglia activation, especially on neuronal death (Fig. 9b). Terminal deoxynucleotidyl transferase dUTP nick end labeling (TUNEL) assay revealed four groups of neurons from the aspect of tau incorporation and neuronal death (Fig. 9b). In nonpolymerized Tau 410 injection, cell death was significantly increased in neurons that did not incorporate injected tau (Taunegative neurons); this increase was suppressed by PQBP1binding mutations of tau (Fig. 9b, left graphs). Consistently, the increase of cell death in Tau-negative neurons was suppressed by tamoxifen-induced Pqbp1-cKO (Fig. 9b, first line right graph). The effect of Tau 410 on Tau-negative neurons was also suppressed by tau polymerization (Fig. 9b, first line left graph), consistent with the decreased inflammatory gene responses of microglia by tau polymerization (Fig. 3). On the other hand, cell death was increased by tau polymerization in neurons that incorporated tau (Tau-positive neurons), which was not suppressed by PQBP1-binding mutations of tau (Fig. 9b, left graphs) or by PQBP1 depletion (Fig. 9b, right graphs).

In contrast to the in vivo effect in the brain where neurons and microglia co-existed, Tau 410 addition to primary neuron culture revealed that non-polymerized Tau 410 induced no neuronal death in the absence of microglia (Fig. 9c). Meanwhile, neuronal cell death tended to increase following the incorporation of polymerized Tau 410 but not significantly (Fig. 9c). All of these results suggested that tau monomer-induced and PQBP1dependent activation of microglia indirectly induced cell death of Tau-negative neurons, and that incorporation of polymerized tau to neurons directly induced cell death to a lesser extent (Fig. 9d). The indirect pathway via microglia is mediated by cytokines such as TNF, ISG54, CXCL10, and IFN $\beta$ in LPLnegative microglia. Meanwhile, LPL-positive microglia around $A \beta$ plaques might inhibit tau seeding ${ }^{63}$, and LPL-negative microglia in the absence of $\mathrm{A} \beta$ plaques might somehow contribute to tau aggregate seeding 67 .

FTLD-linked disease mutation of Tau does not affect PQBP1dependent microglial activation. To elucidate the pathological meaning of FTLD-linked disease mutation of tau (P301S) ${ }^{68,69}$ in PQBP1-mediated microglia activation, we compared Tau 441 and Tau 441 P301S regarding affinity to PQBP1, induction of nuclear translocation of NFKB, gene expression of inflammation genes, and direct effect on cell death in primary neuron culture (Supplementary Fig. 9). Their affinities to PQBP1 were equivalent in SPR (Supplementary Fig. 9a and Supplementary Table 2); they were similarly taken up by primary microglia to merge with PQBP1 at cytoplasmic foci (Supplementary Fig. 9b); their dynamics for merging with PQBP1 were similar even if they were polymerized (Supplementary Fig. 9c, d); they similarly induced nuclear translocation of $\mathrm{NF \kappa B}$ in primary culture microglia, which were similarly decreased by polymerization (Supplementary Fig. 9e); they similarly upregulated Tnf and Isg54 gene expression in RT-PCR of primary microglia, which were similarly suppressed by polymerization (Supplementary Fig. 9f); in immunohistochemistry of mouse brain, they were similarly colocalized with PQBP1 and cGAS at cytoplasmic foci of microglia (Supplementary Fig. 9g); and they similarly induced nuclear translocation of NFKB in brain microglia, which was equally suppressed by tamoxifen-induced Pqbp1-cKO (Supplementary Fig. 9g). Although these data indicated similar PQBP1dependent microglial activation by Tau 441 and Tau 441 P301S, induction of pro-inflammatory genes was more prominent by Tau 441 P301S than Tau 441 (Supplementary Fig. 9h).

TUNEL staining of the injected mice revealed enhancement of neuronal cell death by Tau P301S mutation and polymerization (Supplementary Fig. 9i), which was independent of PQBP1 deficiency in microglia of tamoxifen-induced Pqbp1-cKO (Supplementary Fig. 9i). As expected, Tau P301S incorporation directly induced such neuronal cell death in primary neurons without microglia (Supplementary Fig. 9j).

The discrepancy between the effects of Tau 441 P301S on microglia in vitro and in vivo for induction of pro-inflammatory genes could be explained by non-cell autonomous factors originated from dying neurons that should release various factors including DAMPs like HMGB1 and $A \beta^{4,70-72}$ and senescenceassociated secretary phenotype factors (SASPs) ${ }^{73}$.

PQBP1 in microglia as a possible therapeutic target. Finally, we examined how normal form, binding mutants and disease mutant of tau in non-polymerized or polymerized state affect mouse memory functions after their injection into bilateral entorhinal cortex (Fig. 7a) by using the Y-maze test for short-term memory and the Morris water maze test for long-term memory (Fig. 10a). In non-polymerized state, both tests revealed that Tau 410 strongly, but Tau 441 and Tau 441 P301S weakly, impaired memory functions (Fig. 10a, upper graphs). The impairment was recovered when Tau 410 possessed mutations for binding to PQBP1 (Fig. 10a, upper graphs). Impairment of memory by Tau 410 and Tau 441 was also recovered when PQBP1 was depleted in microglia, while the effect of Tau 441 P301S was resistant to microglial PQBP1 depletion (Fig. 10a, upper graphs). These results of memory functions were consistent with the in vivo effects of tau species on neuronal death and their modification by microglial PQBP1 depletion (Fig. 10b and Supplementary Fig. 9i).

On the other hand, when tau species were polymerized (Fig. 10a, lower graphs), Tau 441 impaired memory functions especially when it had the disease-linked mutation (Fig. 10a, lower graphs), though Tau 410 and their PQBP1-binding mutants did not affect memory function (Fig. 10a, lower graphs). Together with their effects on neuronal cell death in vivo (Fig. 9b, Supplementary Fig. 9i) and their effect on neuronal cell death in vitro (Fig. 9c, Supplementary Fig. 9j), the memory phenotypes could be explained by direct toxicities of Tau 441 and Tau 441 P301S taken up by neurons that were higher than Tau 410 or the PQBP1-binding mutants. 
a

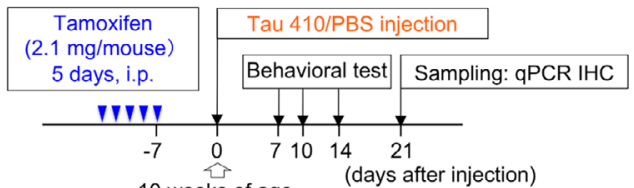

b
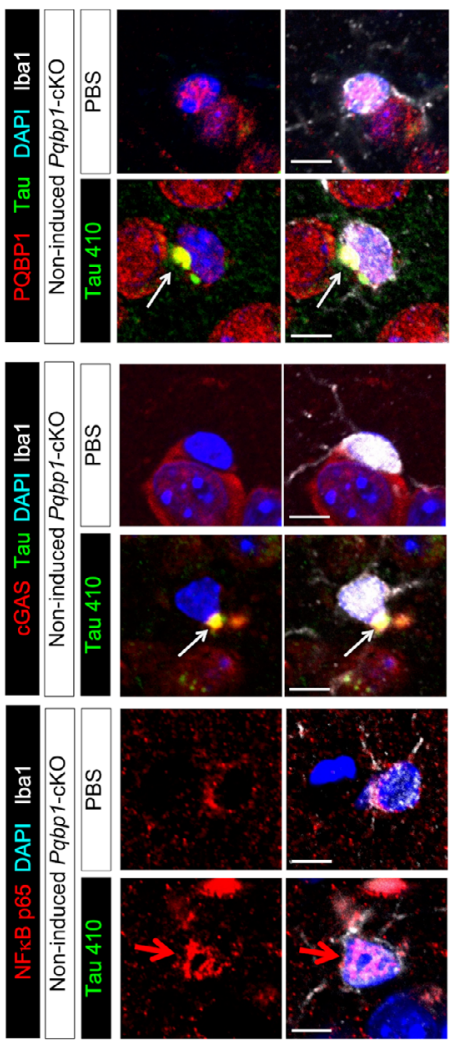

e

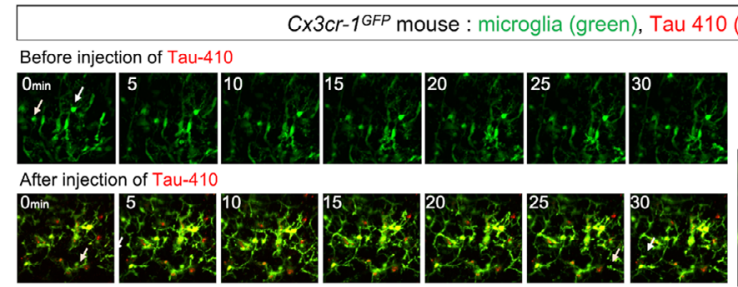

Cx3cr-1 ${ }^{\text {GFP }}$ mouse + siPQBP1 : microglia (green), Tau 410 (red)
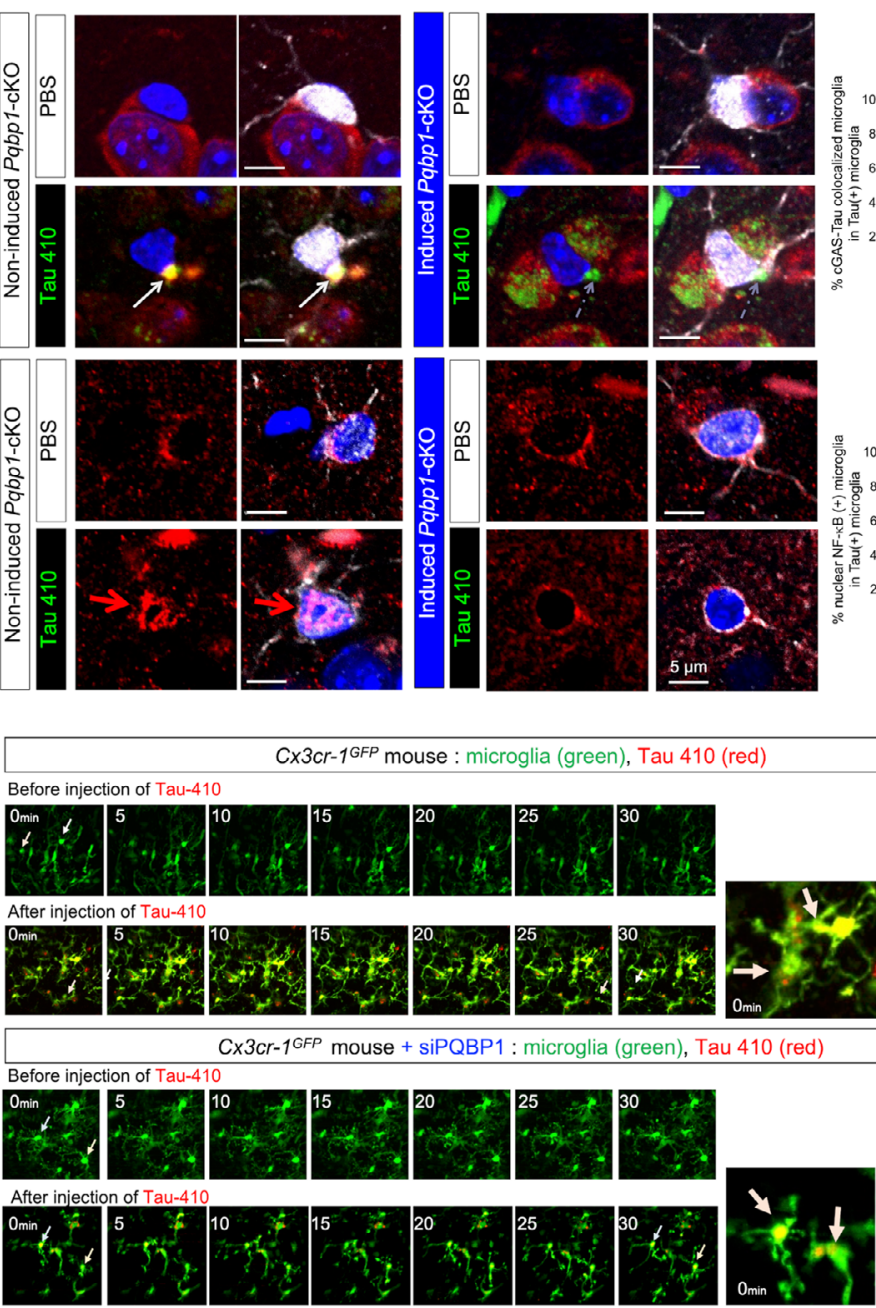

\section{4 groups of Pqbp1-cKO + tamoxifen / Tau}

Group 1: Pqbp1-cKO without tamoxifen + PBS

Group 2: Pqbp1-cKO without tamoxifen + Tau410

Group 3: Pqbp1-cKO with tamoxifen + PBS

Group 4: Pqbp1-cKO with tamoxifen + Tau410

c

d

$N=5$. \#P: $P<0.01$
in Tukey's HSD test

\#\#: $P<0.01$ in Tukey's HSD test Tnf

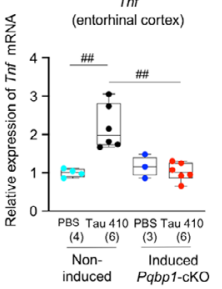

$\begin{gathered}\text { PBS Tau410 PBS Tau410 } \\ \text { Non- } \\ \text { induced }\end{gathered}$
$\begin{gathered}\text { Induced } \\ \text { Pqbpt-cKO }\end{gathered}$
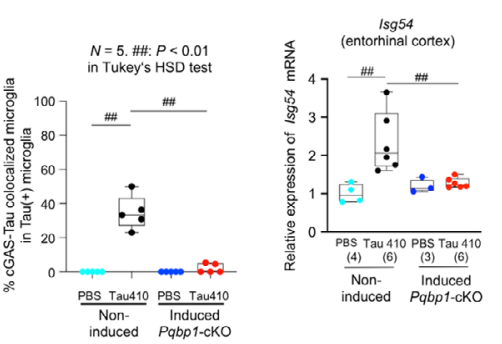

Y-maze test

\#P $P$ : $0.01, \#: P<0.05$

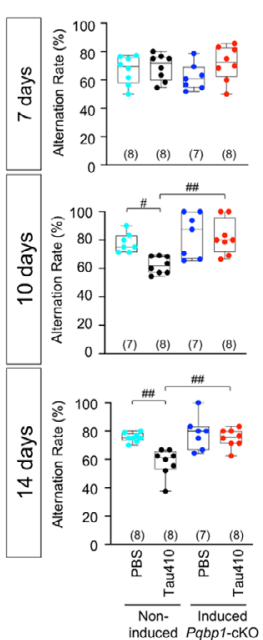

$N=5$, \# $P<0.01$
in Tukey's HSD test
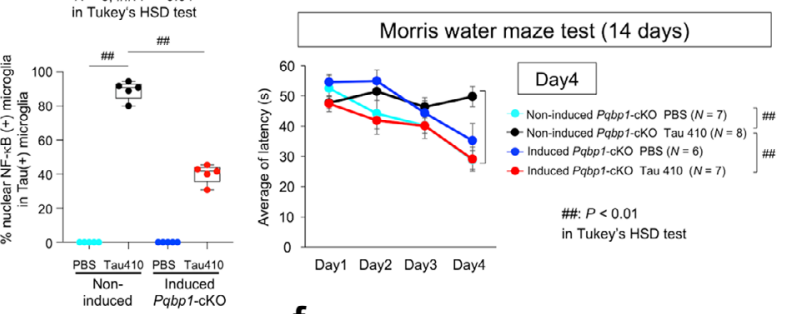

$\mathbf{f}$

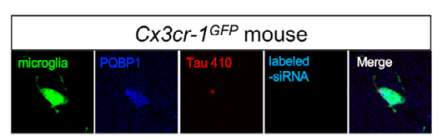

Cx3cr-1 GFP mouse + siPQBP1
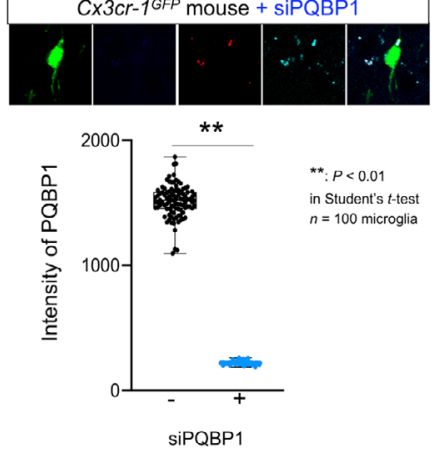

Collectively, our results indicate that tau activates microglia by tau monomer-induced PQBP1-dependent pathway (LRP1-PQBP1 pathway) and PQBP1-independent pathway (TREM2-ERK/PI3K pathway) that trigger neuronal death in parallel (Fig. 10b, left panels). Conversely, neuronal cell death directly induced by incorporation of tau polymer (especially of disease mutant tau) triggered microglia activation (Fig. 10b, right panels). The vicious circle generated by activated microglia and damaged neurons based on critical molecules, including LRP1, TREM2, and PQBP1, would definitely contribute to AD and tauopathy pathologies and would be critical therapeutic targets.

\section{Discussion}

This study revealed that the cGAS-STING pathway works similarly both in virus infections and neurodegenerative diseases (Supplementary Fig. 10). Unexpectedly, intracellular dynamics of tau protein such as direct incorporation into rough ER was also homologous to that of corona viruses including SARS-CoV-2 of COVID-19 (refs. ${ }^{46,47}$ ) (Supplementary Fig. 10). Moreover, results obtained in this study have provided us with a further perspective that tau-propagation-induced pathologies of tauopathy are based not only on prionoid propagation among neurons, which possesses a kind of specificity in propagation pattern ${ }^{38-40}$, but also 
Fig. 6 PQBP1 is essential for in vivo activation of microglia by extrinsic Tau. a Protocol of Tau 410 monomer $(2.2 \mu \mathrm{g} / \mu \mathrm{l}, 1 \mu \mathrm{l} / \mathrm{injection}, 4.4 \mu \mathrm{g} / \mathrm{mouse})$ or equivalent PBS injection to bilateral entorhinal cortices of non-induced or tamoxifen-induced Pqbp1-cKO mice. Four groups were made for analyses. i.p. intra-peritoneal injection, qPCR quantitative RT-PCR, IHC immunohistochemistry. b Immunohistochemistry of Iba1-positive microglia with PQBP1-Tau colocalization, cGAS-tau colocalization, and NFKB nuclear translocation in injected areas of non-induced Pqbp1-cKO and tamoxifen-induced Pqbp1-cKO. (Right) Quantitative analyses of PQBP1-Tau colocalized microglia, cGAS-tau colocalized microglia, and activated microglia (nuclear NFkB-positive) among

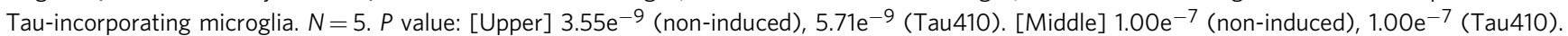
[Lower] 2.29 $\mathrm{e}^{-14}$ (non-induced), 6.98 $\mathrm{e}^{-12}$ (Tau410). c RT-qPCR of induction of Tnf and Isg54 by Tau 410 in entorhinal cortex from non-induced or tamoxifen-induced Pqbp1-cKO mice. $N=(4,6,3,6) . P$ value: [Upper] 0.0011 (non-induced), 0.0005 (Tau410). [Lower] 0.0031 (non-induced), 0.0080 (Tau410). d Y-maze test at 7, 10, and 14 days and Morris water maze test at 14 days after tau injection into bilateral entorhinal. Data points (Morris water maze test): mean \pm SEM. Y-maze: $N=(8,8,7,8)$ for 7 days, $(7,8,7,8)$ for 10 days and $(8,8,7,8)$ for 14 days. Morris water maze: $N=(7,8,6,7)$. $P$ value: [Y-maze; 10 days] 0.0439 (non-induced), and 0.0055 (Tau410). [14 days] 0.0014 (non-induced), and 0.0018 (Tau410). [Morris water maze, Day4] 0.0043 (non-induced), and 0.0048 (Tau410). e In vivo live imaging of microglia by two-photon microscopy with B6.129P2(Cg)-Cx3crtmlitt/J (Cx3cr-1 GFP) mice in which microglia were labeled by the endogenous $\mathrm{Cx} 3 \mathrm{cr}$ 1 enhancer/promoter-driven EGFP protein. $\mathbf{f}$ Immunohistochemistry confirmed PQBP1-KD by siRNA-PQBP1 injection. (Graph) Quantification of PQBP1 signal intensity/cell. Data points: mean \pm SEM. $n=100$ microglia. $P=1.71 e^{-102, \star \star} P<0.01$ in twosided Student's t-test. Box plots show the median, quartiles, and whiskers that represent data outside the 25th to 75th percentile range. Tuple of $N$ numbers indicates PBS/non-induced, Tau410/non-induced, PBS/induced Pqbp1-cKO, and Tau410/induced Pqbp1-cKO), respectively. For multiple group comparisons, Tukey's HSD test was performed ( $\left.{ }^{\#} P<0.01,{ }^{\#} P<0.05\right)$.

on substance diffusion between neurons and microglia, which by itself does not define a specificity for expansion pattern in the brain.

PQBP1 was the firstly identified intracellular receptor for HIV in the cGAS-STING pathway ${ }^{8}$, which was followed by the discovery of various intracellular receptors such as ZCCHC3 (ref. ${ }^{9}$ ) and G3BP1 (ref. ${ }^{10}$ ) for extrinsic DNAs. Meanwhile, since these proteins have also protein-protein interactions, if the intracellular receptors include neurodegenerative disease proteins such as PQBP1, the cGAS-STING pathway in microglia might act similarly to extrinsic viruses ${ }^{74}$ and to extrinsic neurodegenerative disease proteins, as hypothesized previously ${ }^{37}$. Given that the hypothesis was proven by this study, the scheme could be further expanded to huntingtin, ataxin-1 and ataxin-3 via PQBP1 (refs. 12,13,41) and possibly to other target neurodegenerative proteins via certain intracellular receptors in the cGAS-STING pathway that may be discovered in the future. Interestingly, human FUS, TDP43, hnRNPA1, and hnRNPA2B1, which cause amyotrophic lateral sclerosis (ALS) ${ }^{75}$, contain multiple prolines (https://www.uniprot.org/), and mutations around which are related to the severity of disease $\mathrm{e}^{76}$.

The second issue raised by this study could also be considered as the relationship between intercellular transmission among neurons and one-way transmission of tau proteins to microglia. Tau and some other neurodegenerative disease proteins propagate in the brain, at least in cell and animal model experiments ${ }^{38-40}$. The mechanism of how extracellular tau proteins are taken up into neurons is not exactly known, while fundamental mechanism such as phagocytosis, pinocytosis, receptor-mediated uptake, exosome-ectosome, nanotube, and so on are suggested 77 . Endocytosis, including phagocytosis, pinocytosis, and receptor-mediated uptake ${ }^{78}$, is fundamentally shared between microglia and neurons. Our experiments showed that the responses of microglia to different isoforms of tau such as $4 \mathrm{R}$-tau (Tau 441) and 3R-tau (Tau 410) are similar, in contrast to interneuronal prionoid transmission patterns specific to tau species ${ }^{49,79}$. Intriguingly, the cGAS-STING system does not require aggregation of disease proteins, which is templatedirected misfolding/aggregation and dependent on difference of isoforms/species ${ }^{49,79}$.

Genetic studies have revealed that TREM2 is a risk factor of $\mathrm{AD}^{80,81}$, and TREM2 mediates $\mathrm{A} \beta$-sensing of microglia ${ }^{82}$. A comprehensive single cell RNA-seq study revealed a diseasespecific group of microglia, which are activated by TREM2dependent and TREM2-independent mechanisms in mouse AD model and human $\mathrm{AD}$ patient brains ${ }^{51}$. In addition, such a group of disease-associated microglia takes up $\mathrm{A} \beta$ to intracellular/phagocytic particles ${ }^{51}$. Therefore, an important question would be whether the PQBP1-cGAS-STING pathway-dependent activation of microglia by non-aggregated tau revealed in this study corresponds to the TREM2-independent activation of microglia. Presumably but not exclusively LPL, a marker of the disease-specific microglia ${ }^{51}$, was expressed in microglia whose activation was mediated by PQBP1 (Supplementary Fig. 8). TREM2 signaling also leads to NFKB activation ${ }^{83}$ and our data revealed that deficiency of PQBP1-cGAS-STING pathway leads to 50\% suppression of NFKB activation (Figs. 4 and 5), supporting the hypothesis that the PQBP1-cGAS-STING pathway induced by tau and TREM2-pathway induced by A $\beta$ are complementary mechanisms involved in the activation of microglia and their conversion to disease-specific microglia in AD brains. Consistently, our genetic analysis of double KO of Pqbp1 and Lrp1 or Trem2 in microglia by using Luciferase and RFP assays to evaluate their effects (Fig. 8b-d) indicated that LRP1-PQBP1 and TREM2-ERK/PIK3 pathways transduced two lines of cell signaling from tau to NFKB (Fig. 8e).

Our results collectively revealed the monomer-dominant but 3R/4R-equivalent tau effect on PQBP1-mediated microglia activation (Figs. 1-7), which indirectly induced neuronal cell death (Fig. 9 and Supplementary Fig. 9). In addition, we revealed polymer-dominant and 4R/disease-mutant dominant tau effects that directly induced neuronal cell death after their incorporation into neurons (Fig. 9 and Supplementary Fig. 9). Our analysis of their effects on cognitive functions (Fig. 10a), although it was performed under a restrictive condition regarding time window, spatial distribution of pathology, and tau dose dependency, suggested that PQBP1 largely contributes to the indirect pathway while less remarkably to the direct pathway. We previously reported that SRRM2 phosphorylation-induced PQBP1 deficiency in the nucleus causes synapse reduction and cognitive impairment ${ }^{35}$. In this regard, it remains possible that incorporated tau causes PQBP1 dysfunction though an abnormal interaction (Fig. 10b), and the question should be investigated further in the future.

The results of this study indicated that microglia-specific depletion of PQBP1 could be used to develop therapeutics against tau-mediated neurodegenerative diseases. Meanwhile, we have previously shown that impairment of PQBP1 in neurons leads to synaptic loss in the early stage of $\mathrm{AD}$, and adeno-associated virusPQBP1 (AAV-PQBP1) effects could be developed for therapeutics ${ }^{35}$. The current and previous findings could be coordinated since neuronal and microglial functions of PQBP1 
a

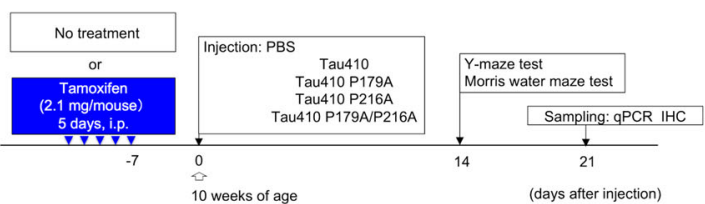

b
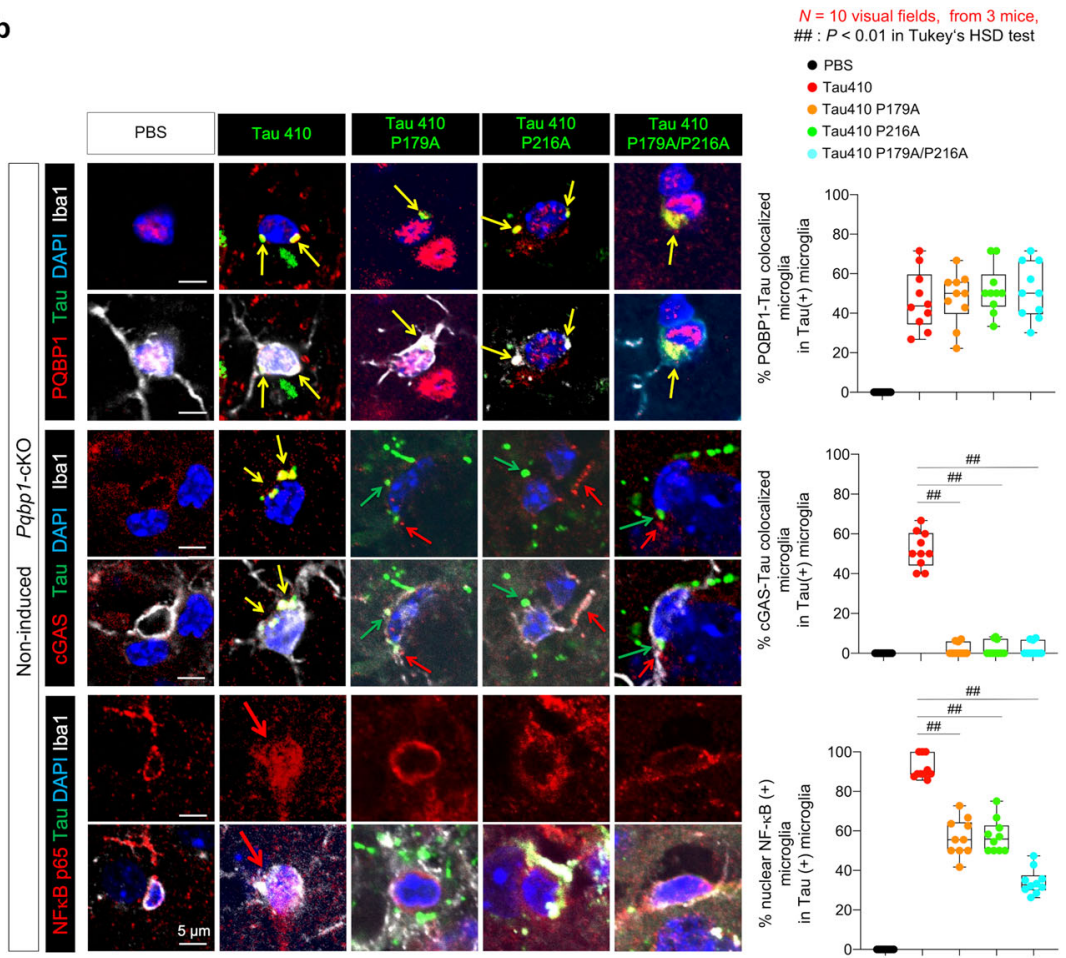

d
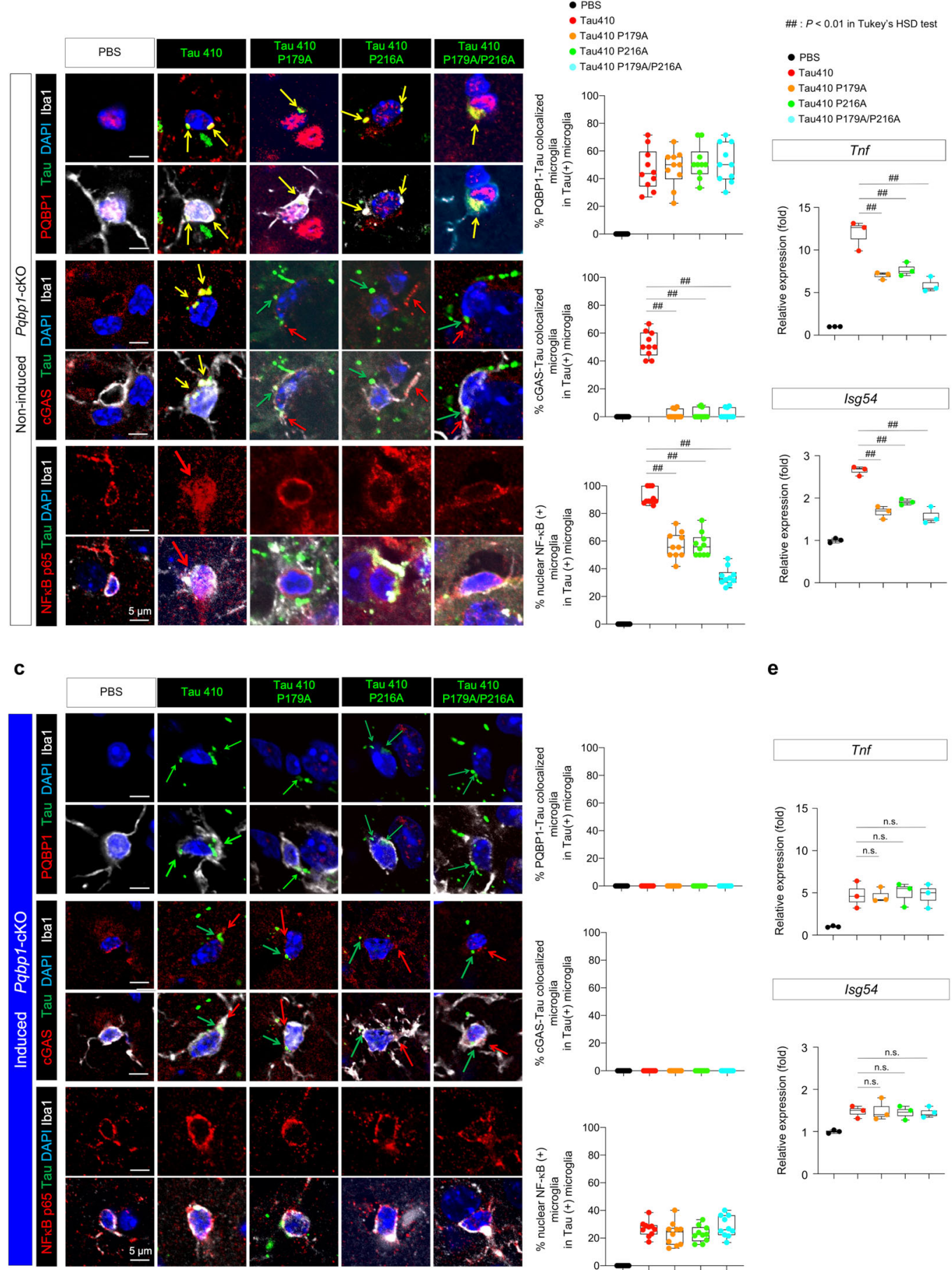

e
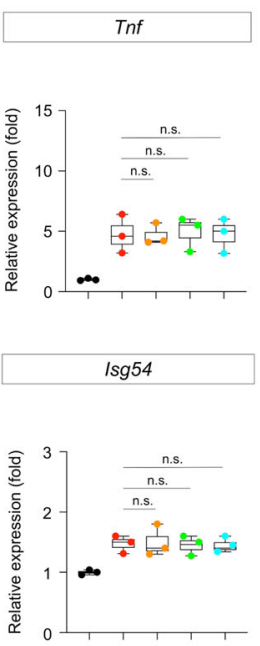

are naturally different. Since our western blot data indicated that AAV-PQBP1 upregulated PQBP1 protein in primary neurons but not in primary microglia (Supplementary Fig. 11), AAV-PQBP1 also remains as a seed therapy for $\mathrm{AD}^{35}$ in addition to the microglia-specific $\mathrm{KO}$ or knockdown by utilizing anti-sense oligonucleotide, gene therapy, genome editing, and other newest techniques.
We observed tamoxifen-inducible microglia-specific homozygous Pqbp1-cKO mice for 6 months after administration of tamoxifen. These mice did not show any abnormality in their motor activity, eating, appearance, or behavior, though they were kept in the SPF condition. This might be because microglia activation is partially by the PQBP1-independent system (Fig. 8e). If PQBP1 deficiency in microglia is similarly safe for human 
Fig. 7 PQBP1-Tau interaction is essential for in vivo activation of microglia. a Protocol of injection of Tau 410 or Tau 410 PQBP1-binding mutant monomers $(0.5 \mu \mathrm{g} / \mu \mathrm{l}, 4.0 \mu \mathrm{l} /$ injection, $4.0 \mu \mathrm{g} /$ mouse) or equivalent PBS to bilateral entorhinal cortices of non-induced or tamoxifen-induced Pqbp1-cKO mice. Five groups were used for analyses. b, c Immunohistochemistry of Iba1-positive microglia with PQBP1 + tau, cGAS+tau, NFkB-p65+tau colocalization in injected area of non-induced Pqbp1-cKO, and tamoxifen-induced Pqbp1-cKO. Quantitative analyses of PQBP1-Tau colocalized microglia, cGAS-tau colocalized microglia, and activated microglia (nuclear NFkB-positive) among Tau-incorporating microglia are shown in the graphs on the right. $N=10$ (visual fields, from 3 mice). $\# P<0.01$ in Tukey's HSD test. d, e RT-qPCR of Tnf and Isg54 induction by Tau 410 and the PQBP1-binding mutants with entorhinal cortex tissues prepared from non-induced or tamoxifen-induced Pqbp1-cKO mice. $N=3$. \#\# $P<0.01$, n.s. $P>0.05$ in Tukey's HSD test (twosided, multiple comparison). Box plots show the median, quartiles, and whiskers that represent data outside the 25th to 75th percentile range.

a

b
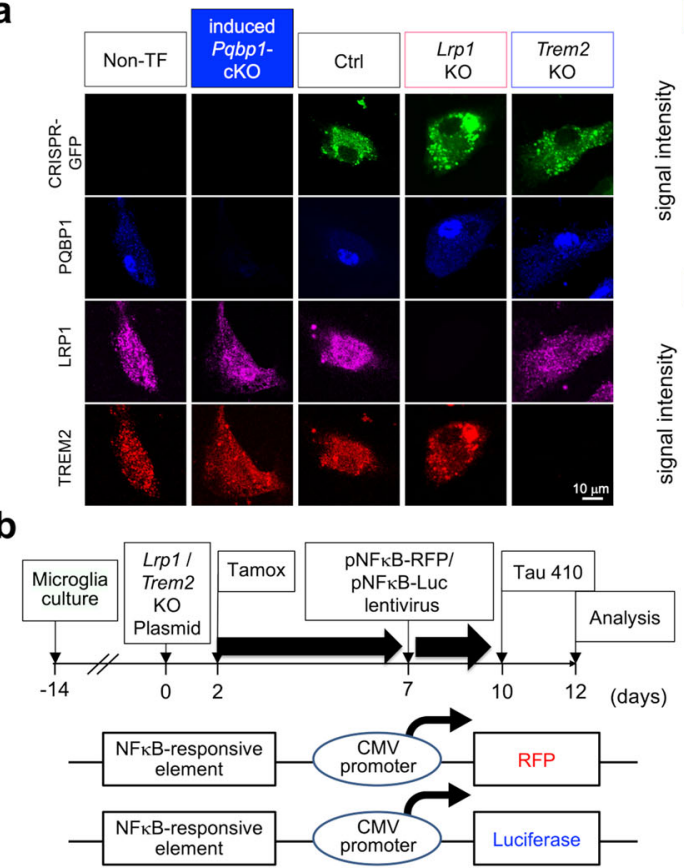

d
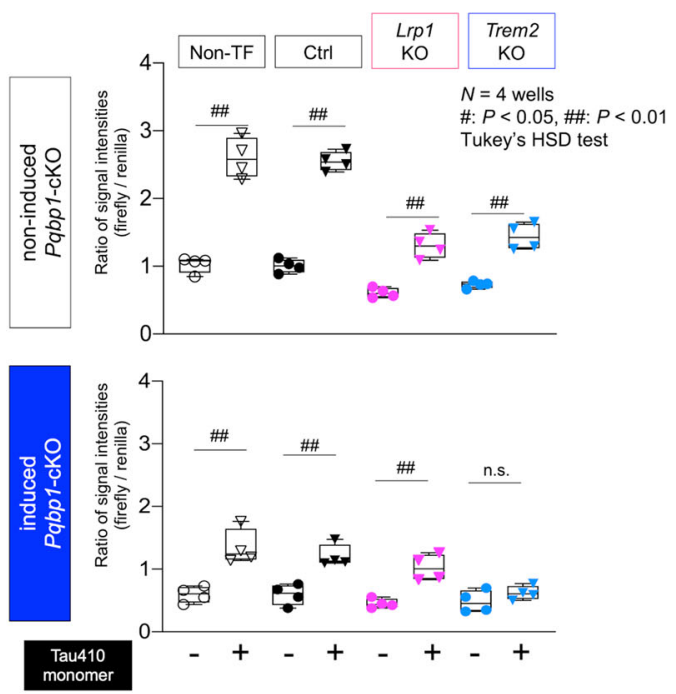

e

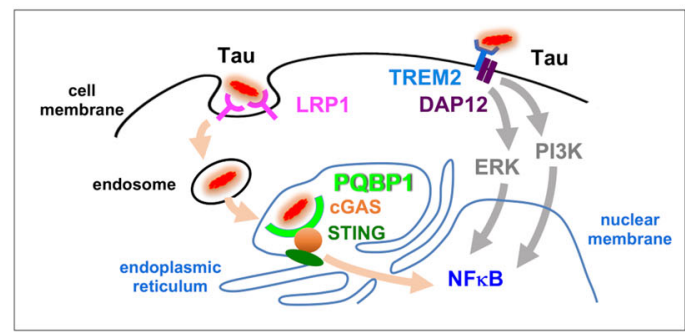

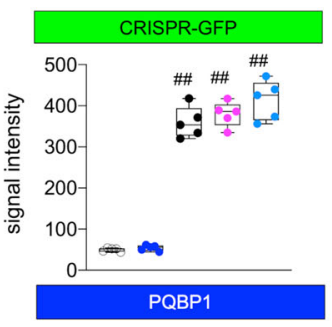
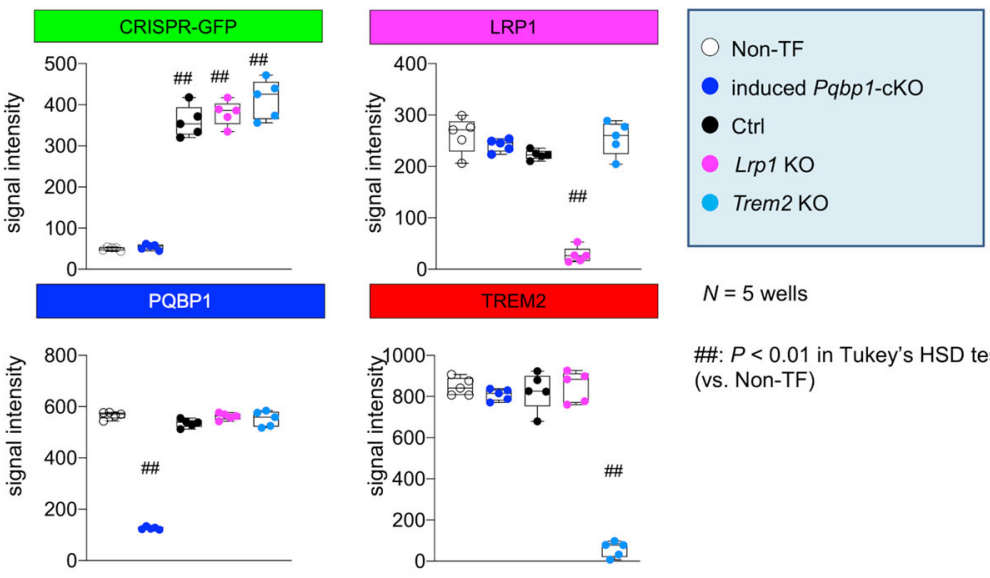

$N=5$ wells

\#\#: $P<0.01$ in Tukey's HSD test (vs. Non-TF)

c
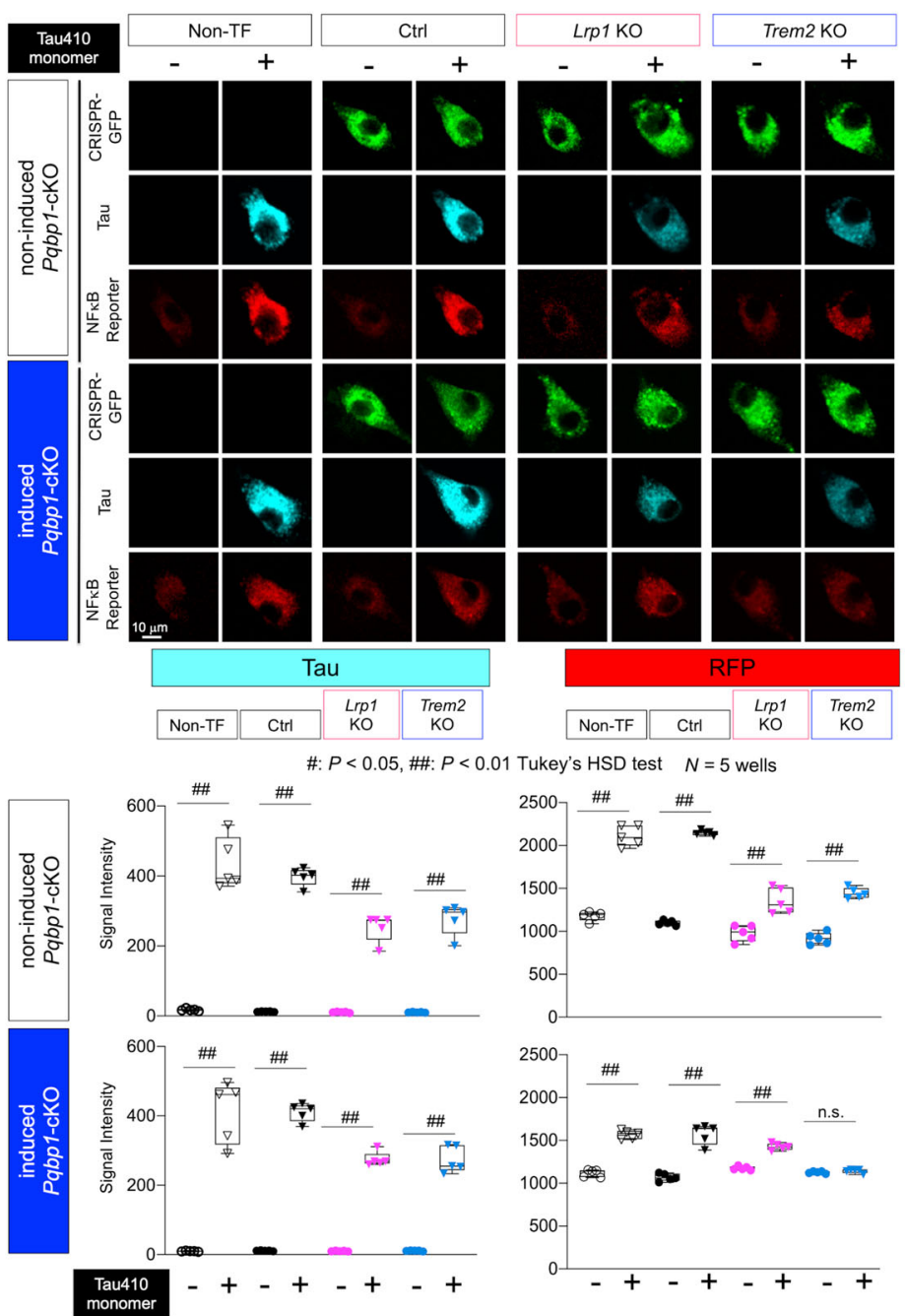
Fig. 8 Genetic interaction of PQBP1 with LRP1 or TREM2 in microglia. a Immunocytochemistry revealed high efficiency of tamoxifen-induced Pqbp1cKO and D10A-mutant Cas9 nuclease/sgRNA-mediated KO of Lrp1/Trem2. Right graphs show quantitative analyses of signal intensities of target genes. Non-TF non-transfected. $N=5$ wells. In the analysis of CRIPER-GFP signals: $P=7.35 \mathrm{e}^{-12}$ (Ctrl vs Non-TF), $2.22 \mathrm{e}^{-12}$ (Lrp1-KO vs Non-TF), $3.31 \mathrm{e}^{-13}$ (Trem2-KO vs Non-TF); in the analysis of LRP1 signal: $P=1.09 \mathrm{e}^{-11}$ (Lrp1-KO vs Non-TF); in the analysis of PQBP1 signals: $P=1.92 \mathrm{e}^{-14}$ (Pabp1-cKO vs Non-TF); in the analysis of TREM2 signals: $P=6.76 \mathrm{e}^{-14}$ (Trem2-KO vs Non-TF); \#\# $P<0.01$ in Tukey's HSD test (vs Non-TF). b Protocol for double KO of Pqbp1-Lrp1 or Pqbp1-Trem2 genes in primary microglia and their activation of NFkB by Tau 410. Tamox tamoxifen. c RFP signal assay to monitor activation of NFKB by adding Tau 410 in single KO microglia of Lrp1 or Trem2 or double KO microglia of Pqbp1-Lrp1 or Pqbp1Trem2. $N=5$ wells. In tau signal assay of non-induced Pabp1-cKO group: $P=1.002 \mathrm{e}^{-13}$ [Non-TF (Tau+) vs (Tau-)], $1.005 \mathrm{e}^{-13}[\mathrm{Ctrl}(\mathrm{Tau}+)$ vs (Tau $-)], 5.65 \mathrm{e}^{-11}[\mathrm{Lrp1}-\mathrm{KO}(\mathrm{Tau}+)$ vs $(\mathrm{Tau}-)], 4.597 \mathrm{e}^{-12}[$ Trem2-KO (Tau + ) vs $(\mathrm{Tau}-)]$; in tau signal assay of induced Pqbp1-cKO group: $P=1.005 \mathrm{e}$ $-13[$ Non-TF $(\mathrm{Tau}+)$ vs $(\mathrm{Tau}-)], 1.006 \mathrm{e}^{-13}[\mathrm{Ctrl}(\mathrm{Tau}+)$ vs $(\mathrm{Tau}-)], 1.73 \mathrm{e}^{-11}\left[\right.$ Lrp1-KO (Tau+) vs (Tau-)], 1.98 $\mathrm{e}^{-11}[$ Trem2-KO (Tau+) vs (Tau $-)$ ]; in RFP signal assay of non-induced Pqbp1-cKO group: $P=4.60 e^{-13}\left[\mathrm{Non}-\mathrm{TF}(\mathrm{Tau}+)\right.$ vs (Tau-)], $1.23 \mathrm{e}^{-13}[\mathrm{Ctrl}(\mathrm{Tau}+)$ vs $(\mathrm{Tau}-)], 4.93 \mathrm{e}^{-7}$ [Lrp1-KO (Tau+) vs (Tau-) ], 1.15e $\mathrm{e}^{-12}\left[\right.$ Trem2-KO (Tau+) vs (Tau-) ]; in RFP signal assay of induced Pqbp1-cKO group: $P=1.005 \mathrm{e}^{-13}[\mathrm{Non}-\mathrm{TF}(\mathrm{Tau}$ + ) vs $(\mathrm{Tau}-)], 1.006 \mathrm{e}^{-13}[\mathrm{Ctrl}(\mathrm{Tau}+)$ vs $(\mathrm{Tau}-)], 1.73 \mathrm{e}^{-11}[$ Lrp1-KO (Tau+) vs (Tau-), $0.9998[$ Trem2-KO (Tau+) vs (Tau-)]; \#P<0.05, $\# \# P<0.01$ in Tukey's HSD test. $\mathbf{d}$ Luciferase assay to monitor activation of NFKB by adding Tau 410 to single KO microglia of Lrp1 or Trem2 or double KO microglia of Pqbp1-Lrp1 or Pqbp1-Trem2. $N=4$ wells. In non-induced Pqbp1-cKO group: $P=2.20 e^{-11}\left[\right.$ Non-TF (Tau+) vs (Tau-)], 3.31e ${ }^{-11}[$ Ctrl $(\mathrm{Tau}+)$ vs $(\mathrm{Tau}-)], 7.40 \mathrm{e}^{-5}[$ Lrp1-KO (Tau+) vs (Tau-) $], 5.76 \mathrm{e}^{-15}\left[\right.$ Trem2-KO (Tau + ) vs (Tau-)]; in induced Pqbp1-cKO group: $P=8.13 \mathrm{e}^{-5}[\mathrm{Non}-$ $\operatorname{TF}(\mathrm{Tau}+)$ vs $(\mathrm{Tau}-)$ ], $0.0013\left[\mathrm{Ctrl}(\mathrm{Tau}+)\right.$ vs $(\mathrm{Tau}-)$ ], $0.0028\left[\right.$ Lrp1-KO $(\mathrm{Tau}+)$ vs $(\mathrm{Tau}-)$ ], $0.9498[$ Trem2-KO $(\mathrm{Tau}+)$ vs $(\mathrm{Tau}-)] ;{ }^{\#} P<0.05$, $\# \#<0.01$ in Tukey's HSD test. e A hypothetical scheme of two pathways from extrinsic tau to activation of nuclear NFkB in microglia. Box plots show the median, quartiles, and whiskers that represent data outside the 25th to 75th percentile range.

patients, especially under the risk of infection, PQBP1 in microglia could be a target of therapeutics against $\mathrm{AD}$, tauopathy, and other neurodegenerative diseases. Similar drug-inducible and microglia-specific cKO or conditional knockdown (cKD) technologies based on a viral vector could be developed.

\section{Methods}

Plasmid construction. The DNAs encoding full-length PQBP1, PQBP1(1-94), Tau 441, and Tau(244-341) of Tau 410 were inserted into NdeI and BamHI sites of a pOPTH plasmid, which was used to express the protein fused to an N-terminal His-tag in E. coli ${ }^{20}$. The DNAs encoding Tau 410, Tau 441, A $\beta 40$, and $\alpha$-synuclein were inserted into NdeI and BamHI sites of a pOPHBL plasmid, which was used to express the protein fused to a sequence containing the N-terminal His-tag, a biotinylation-tag, and a lipoyl domain. The DNAs encoding Tau(1-150),

Tau(151-197), Tau(198-243), and Tau(342-410) of Tau 410 were inserted into $\mathrm{NdeI}$ and BamHI sites of a pOPHLT-s plasmid, which was used to express the proteins fused to a sequence containing an N-terminal His-tag, a lipoyl domain, and a TEV protease cleavage site ${ }^{21}$.

Mutagenesis was carried out with pOPTH -Tau 410. The primer sets for P179A, P216A, P179A/P216A:

(P179A) forward: $5^{\prime}$-cccgcggctaaaacccaccatcctct- $3^{\prime}$,

reverse: $3^{\prime}$-ggttttagccgcgggaggcgttttggc- $5^{\prime}$;

(P216A) forward: $5^{\prime}$-agcctggcaacaccaccgacccgtgaa- $3^{\prime}$,

reverse: $3^{\prime}$-tggtgttgccaggctcggagtacgtga- $5^{\prime}$.

The DNA encoding PQBP1(94-176) and PQBP1(193-265) were inserted into the BamHI and SalI sites of a pGEX6P-1 plasmid ${ }^{18}$. All constructs were verified by DNA sequencing.

A complete list of all primers used in this study has been added to Supplementary Information (Supplementary Table 3).

Protein purification. Rosetta(DE3)pLysS-competent cells (70956, Merck, Darmstadt, Germany) were transformed with pOPTH-Tau 410, pOPTH-Tau 410 P179A, pOPTH-Tau 410 P216A, pOPTH-Tau 410 P179A/P216A, pOPTH-Tau 441, pGEX-6P-1-PQBP1, pGEX-6P-1-PQBP1(1-94), pGEX-6P-1-PQBP1(94-176), pGEX-6P-1-PQBP1(193-265). After the E. coli culture reached an $\mathrm{OD}_{600}$ of 0.5 , IPTG was added at $1 \mathrm{mM}$ and cells were incubated for another $2 \mathrm{~h}$ at $37^{\circ} \mathrm{C}$. The $E$. coli cells were collected, suspended in lysis buffer $\mathrm{H}(20 \mathrm{mM}$ Tris- $\mathrm{HCl} \mathrm{pH} \mathrm{8.0,}$ $100 \mathrm{mM} \mathrm{NaCl}, 20 \mathrm{mM}$ Imidazole, protease inhibitor cocktail (539134, Calbiochem, San Diego, CA, USA)) for His-fusion proteins or in lysis buffer G (20 mM Tris- $\mathrm{HCl}$ $\mathrm{pH} 7.5,100 \mathrm{mM} \mathrm{NaCl}, 1 \mathrm{mM}$ DTT, protease inhibitor cocktail (539134, Calbiochem, San Diego, CA, USA)) for GST-fusion proteins, and sonicated for $8 \mathrm{~min}$. His-fusion proteins were purified by Ni-NTA agarose (30230, Qiagen, Dusseldorf, Germany) and eluted with $8 \mathrm{ml}$ of His elution buffer $(20 \mathrm{mM}$ Tris- $\mathrm{HCl}$ pH 8.0, $100 \mathrm{mM} \mathrm{NaCl}, 500 \mathrm{mM}$ Imidazole, $1 \%$ glycerol, protease inhibitor cocktail (539134, Calbiochem, San Diego, CA, USA)), GST-fusion proteins were purified by glutathione-sepharose 4B (17075601, Cytiva, Marlborough, MA, USA)) and eluted with $2 \mathrm{ml}$ of GST elution buffer (20 mM Glutathion, $50 \mathrm{mM}$ Tris- $\mathrm{HCl} \mathrm{pH} \mathrm{8.0,0.1 \%}$ Triton X-100, protease inhibitor cocktail (539134, Calbiochem, San Diego, CA, USA)).

Surface plasmon resonance analysis. Tau proteins, A $\beta 40$ and $\alpha$-synuclein were fused to an $\mathrm{N}$-terminal His-tag and a biotinylation-tag ${ }^{20}$. SPR measurements were performed with a BIAcore J instrument (GE Healthcare) at $25^{\circ} \mathrm{C}^{20}$. The solution contained $10 \mathrm{mM}$ Tris- $\mathrm{HCl}(\mathrm{pH} 8.5), 150 \mathrm{mM} \mathrm{NaCl}, 3 \mathrm{mM}$ EDTA, and $0.005 \%$ Tween-20.

Tau 410, Tau 441, Tau 410 P179A, Tau 410 P216A, Tau 410 P179A/P216A, or Tau 441 P301S were immobilized on CM5 sensor chips at a density of 1200 RU. Tau 441 P301S protein was purchased from Signal Chem (T08-56GN, Biotech, Richmond, BC, Canada). PQBP1 full-length, PQBP1(1-94), PQBP1(94-176), or PQBP1(193-265) were loaded at multiple doses for $120 \mathrm{~s}$.

NMR analysis. Full-length tau and its fragments were expressed in E. coli BL21 (DE3) grown in $\mathrm{M} 9$ minimal medium supplemented with $15 \mathrm{NH} 4 \mathrm{Cl}$ and $13 \mathrm{C}$ glucose. ${ }^{15} \mathrm{~N}$-labeled and ${ }^{13} \mathrm{C} /{ }^{15} \mathrm{~N}$-labeled proteins were purified with a Ni-NTA agarose resin (Qiagen, Valencia, CA) followed by gel filtration ${ }^{84}$.

All tau proteins were purified by reverse-phase high-performance chromatography. The molecular weights of tau proteins were confirmed by MALDI-TOF mass analyses. The NMR samples contained $0.1-0.5 \mathrm{mM}$ tau, $20 \mathrm{mM}$ sodium phosphate ( $\mathrm{pH} 7.0$ ), $20 \mathrm{mM} \mathrm{NaCl}, 1 \mathrm{mM}$ dithiothreitol, $1 \mathrm{mM} \mathrm{NaN}_{3}, 20$ $\mu \mathrm{M}$ sodium 4,4-dimethyl-4-silapentane-1-sulfonate, and $10 \% \mathrm{D}_{2} \mathrm{O}$. All NMR spectra were recorded on a Bruker Avance- 800 NMR spectrometer with a cryoprobe at an acquisition temperature of $283 \mathrm{~K}$.

Preparation of tau oligomers and fibrils. Tau 410 (T06-54N, Signal Chem, Biotech, Richmond, BC, Canada) and Tau 441 (T08-54N, Signal Chem, Biotech, Richmond, BC, Canada), Tau 410 P179A, Tau 410 P216A, Tau 410 P179A/P216A and Tau 441 P301S (T08-56GN, Signal Chem, Biotech, Richmond, BC, Canada) dissolved in PBS at $2 \mu \mathrm{M}$ containing $0.01 \mathrm{mg} / \mathrm{ml}$ heparin and $2 \mathrm{mM}$ DTT were incubated at $37^{\circ} \mathrm{C}$ with shaking. Incubation was continued for 5 or 10 days to generate tau oligomers or fibrils, respectively.

Quantitative immunoprecipitation. Primary microglia were cultured in the medium containing TAMRA-labeled Tau 410 (SP-502-100, R\&D, Minneapolis, $\mathrm{MN}, \mathrm{USA}$ ) at a concentration of $25 \mathrm{nM}$ for 3 days. Then, microglia were washed with ice-cold PBS, and $7 \times 10^{5}$ cells were harvested with $400 \mu \mathrm{l}$ of lysis buffer (25 mM Tris- $\mathrm{HCl}, 150 \mathrm{mM} \mathrm{NaCl}, 1 \mathrm{mM}$ EDTA, 1\% NP40, 5\% glycerol, and $0.5 \%$ protease inhibitor cocktail). Lysates were rotated for $60 \mathrm{~min}$ at $4{ }^{\circ} \mathrm{C}$ and then centrifuged at $366 \times g$ for $10 \mathrm{~min}$. Supernatants were incubated with $1 \mu \mathrm{g}$ of rabbit anti-PQBP1 (1:80, sc-32910, FL-265, Santa Cruz Biotechnology, Dallas, TX, USA), mouse anti-tau (1:400, MAB361, Merck, Darmstadt, Germany), or human IgG (1:400, 12000C, Thermo Fisher Scientific, Waltham, MA, USA) for $48 \mathrm{~h}$ at $4^{\circ} \mathrm{C}$, and incubated with Protein G Sepharose (GE Healthcare, Buckinghamshire, UK) for $1 \mathrm{~h}$ at $4{ }^{\circ} \mathrm{C}$. Protein $\mathrm{G}$ Sepharose beads were precipitated, washed with lysis buffer three times, and the fluorescence intensity of TAMRA-labeled Tau 410 was measured on a FLUOstar OPTIMA-6 microplate reader (BMG Labtech, Durham, NC, USA) using $540 \mathrm{~nm}$ excitation and $590 \mathrm{~nm}$ emission wavelenghts. For western blot, the samples were suspended in sample buffer $(62.5 \mathrm{mM}$ Tris- $\mathrm{HCl}, \mathrm{pH} 6.8,2 \%$ $(\mathrm{w} / \mathrm{v})$ sodium dodecyl sulfate (SDS), $2.5 \%(\mathrm{v} / \mathrm{v}) 2$-mercaptoethanol, $10 \%(\mathrm{v} / \mathrm{v})$ glycerol, and $0.0025 \%$ (w/v) bromophenol blue), left for $1 \mathrm{~h}$ at $37^{\circ} \mathrm{C}$, and boiled in sample buffer immediately before SDS-polyacrylamide gel electrophoresis (PAGE)

The list of antibodies used in this study with their validations on manufacturers' website has been added to Supplementary Information (Supplementary Table 4). 
a
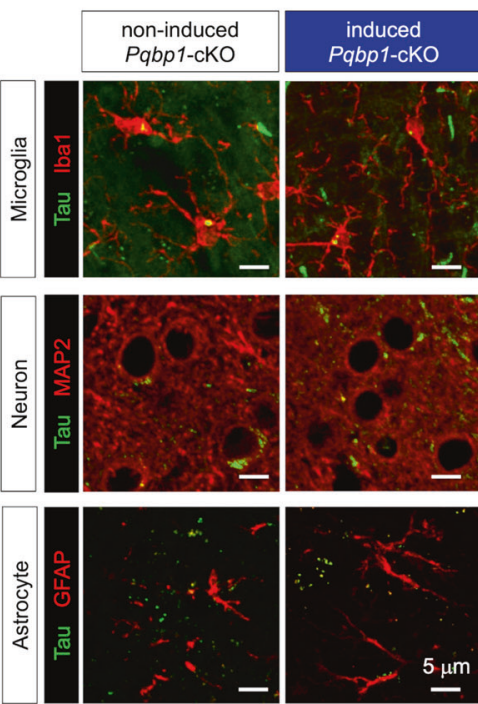

$N=20$ images from 3 mice

non-induced Pqbp 1-cKO

induced Pqbp1-cKO
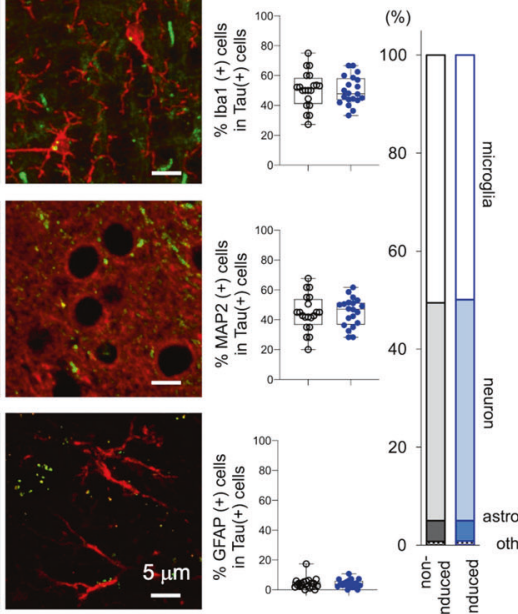

C

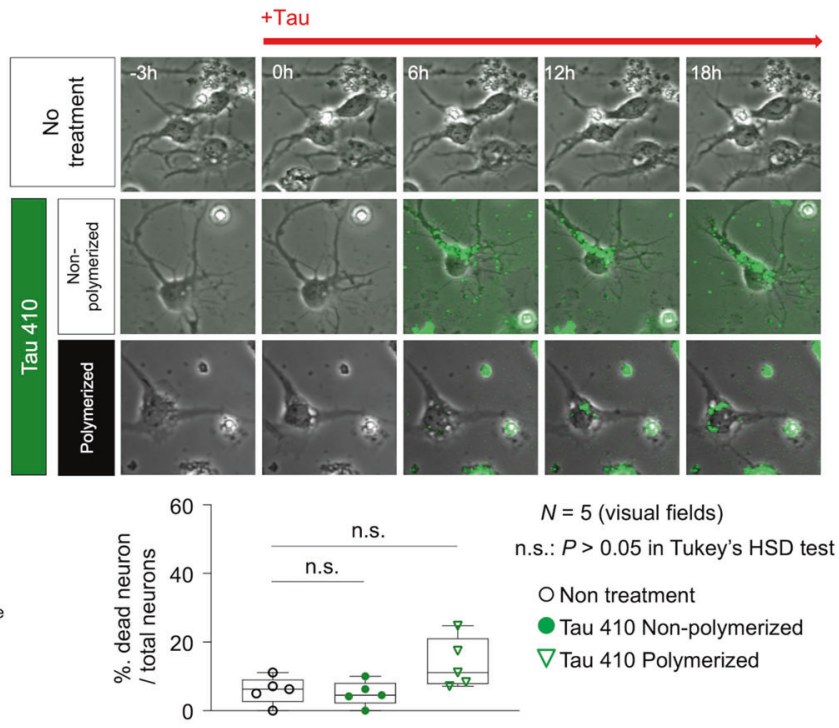

\section{b}

\#: $P<0.01$ \#: $P<0.05$ (comparison among total neurons) \#\#: $P<0.01$, \#: $P<0.05$ (comparison among Tau- neurons) \#: $P<0.01$, \#: $P<0.05$ (comparison among Tau+ neurons)
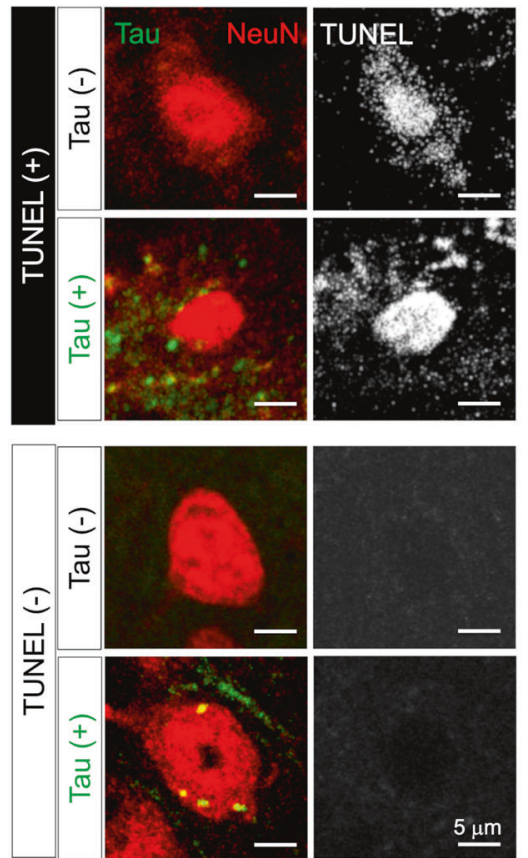

$N=4$ (fields) Tukey's HSD test
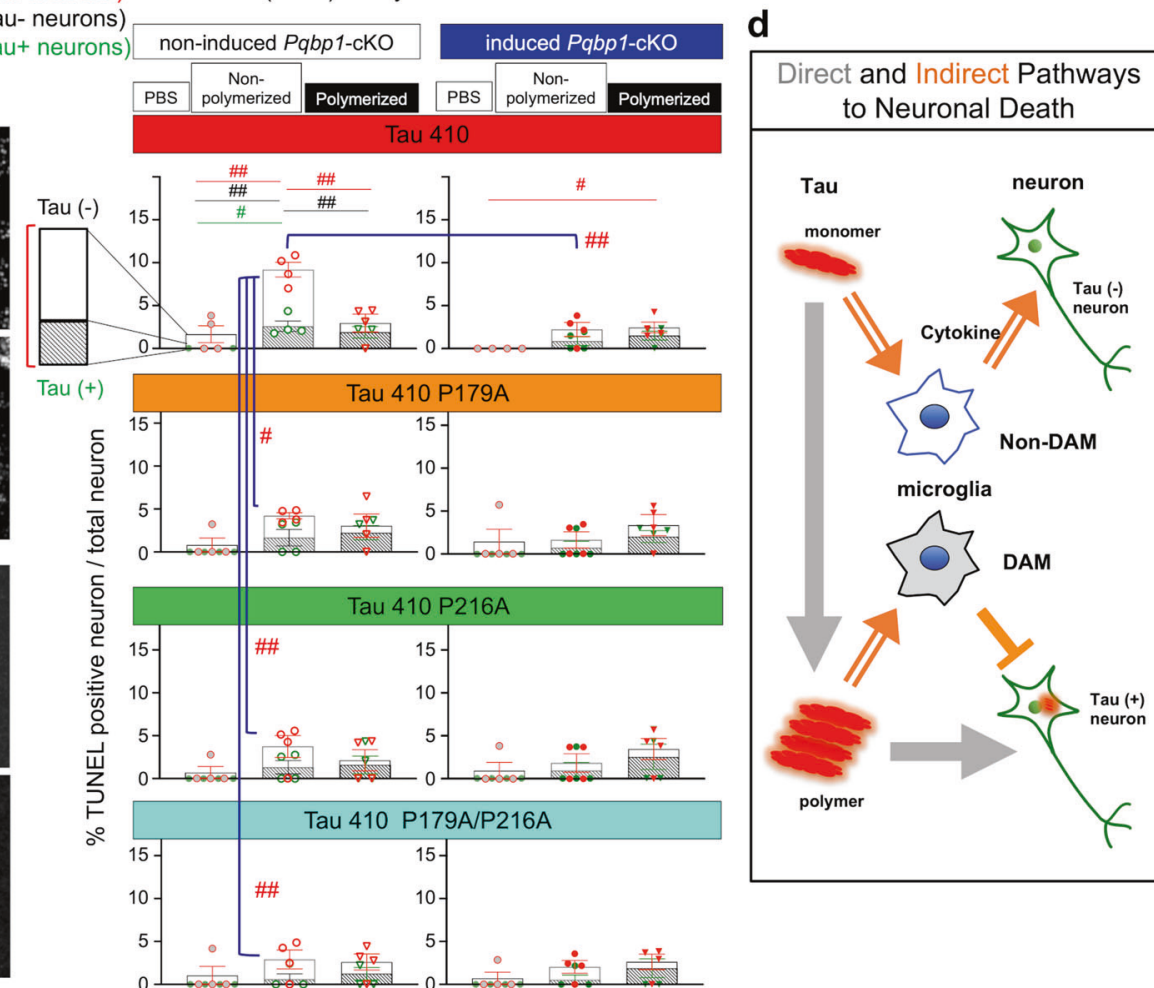

Immunoprecipitation in R6/2 mice. To examine binding of tau and PQBP1 in R6/2 mice, $10 \mathrm{mg}$ of cerebral cortex was lysed by $200 \mu \mathrm{l}$ of RIPA buffer ( $10 \mathrm{mM}$ Tris- $\mathrm{HCl} \mathrm{pH}$ 7.5, $150 \mathrm{mM} \mathrm{NaCl}, 1 \mathrm{mM}$ EDTA pH 8.0, 1\% Triton X-100, 0.1\% SDS, and $0.1 \%$ deoxycholate). The lysate containing $300 \mu \mathrm{g}$ protein was incubated with $1 \mu \mathrm{g}$ of rabbit anti-PQBP1 antibody (1:200, A302-801A, Bethyl, Montgomery, TX, USA) or mouse anti-tau antibody (1:200, ab80579, Abcam, Cambridge, UK) for $2 \mathrm{~h}$ at $4{ }^{\circ} \mathrm{C}$, and incubated with Protein G Sepharose (GE Healthcare, Buckinghamshire, UK) for $2 \mathrm{~h}$ at $4{ }^{\circ} \mathrm{C}$. After centrifugation, the Protein $\mathrm{G}$ Sepharose pellet was washed three times with lysate buffer, boiled at $95^{\circ} \mathrm{C}$ for $10 \mathrm{~min}$, and were subjected to SDS-PAGE.

Generation of Pqbp1-cKO mice. To generate microglia-specific and tamoxifeninducible conditional knockout mouse of Pqbp1 (Pqbp1-cKO) on the C57BL/6 background, tamoxifen-inducible $C \times 3 \mathrm{crl} 1^{\mathrm{CreER} /+}$ male mice were crossed with
$P q b p 1^{f l o x X /+}$ female mice. The double heterozygous mice were further crossed to

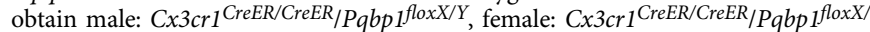

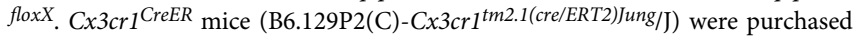
from The Jackson Laboratory (Bar Harbor, ME, USA). Pqbpl-floxed mice were generated as described previously ${ }^{34}$. In all, $100 \mu \mathrm{g} / \mathrm{g}$ tamoxifen $(080 \mathrm{M} 1292 \mathrm{~V}$, Sigma-Aldrich, St Louis, MO, USA) dissolved in corn oil (C8267, Sigma-Aldrich, St Louis, MO, USA) was injected intraperitoneally into Pqbp1-cKO mice once a day for consecutive 5 days to induce depletion of $P q b p 1$ gene in microglia in vivo.

Animal housing conditions. The mice were maintained at $22^{\circ} \mathrm{C}$, under suitable humidity (typically 50\%), and with a 12-h dark/light cycle. Detailed information such as age and sex of the mice is listed in Supplementary Information (Supplementary Table 5). 
Fig. 9 Direct and indirect pathways mediating neuronal toxicity of Tau. a Immunohistochemistry of Tau-injected mice revealed most of the non-microglia cells incorporating tau were MAP2-positive neurons but not GFAP-positive astrocytes. The ratios of Tau-incorporating cells were equivalent in noninduced and tamoxifen-induced Pqbp1-cKO mice, as the basis for analyses in (b). $N=20$ images from three mice. Statistical test: Student's $t$-test. b Frequencies of neuronal cell death (TUNEL-positive and NeuN-positive dying neurons) by addition of normal or PQBP1-binding mutant Tau 410 were changed in Tau-negative neurons in addition to that of Tau-positive neurons. Indirect effect of Tau 410 monomer on Tau-negative neurons was suppressed by PQBP1-binding mutation or by tamoxifen-induced Pqbp1-cKO, while the direct effect of Tau 410 polymers on Tau-positive neurons was not affected by PQBP1-binding mutation or by tamoxifen-induced Pqbp1-cKO. TUNEL terminal deoxynucleotidyl transferase-mediated dUTP nick end labeling. Values in each group are presented as mean \pm SEM. $N=4$ fields. ${ }^{P} P<0.05, \# \# P<0.01$ in Tukey's HSD test. c Direct effect of monomer and polymerized Tau 410 on primary neurons. Non-polymerized Tau 410 was not toxic to neurons in the absence of microglia. $N=5$ visual fields. ${ }^{\text {n.s. }} P>0.05$ in Tukey's HSD test. $\mathbf{d}$ Direct and indirect pathways from extracellular tau to neuronal damage. Tau polymer directly damages neurons, while tau monomer is incorporated into LPL-negative non-DAM microglia (Supplementary Fig. 8), and indirectly damages neurons via cytokines. Though not included in this study, DAM around $A \beta$ plaques might play a different role to prevent tau seeding. Box plots show the median, quartiles, and whiskers that represent data outside the 25 th to 75th percentile range.

Preparation and primary culture of microglia. Mouse primary microglia were prepared from Pqbp1-cKO mice at P1-P3. Cortices were dissected, minced, and digested with $0.05 \%$ Trypsin-EDTA (\#25200056, Thermo Fisher Scientific Inc., MA, USA) at $37^{\circ} \mathrm{C}$ for $10 \mathrm{~min}$. Trypsinization was stopped by adding $10 \%$ fetal bovine serum, and $10 \mathrm{mg} / \mathrm{ml}$ DNase was added to digest the sticky DNA. The cells were collected by centrifugation at $600 \times g$ for $3 \mathrm{~min}$, dissociated by pipetting and passage through a $40 \mu \mathrm{m}$ cell strainer (\#22-363-548, Thermo Fisher Scientific Inc., MA, USA), and seeded into a T-75 culture flask in primary microglia culture medium (MGC57, Cosmo Bio Co., Ltd, Tokyo, Japan) supplemented with $1 \%$ penicillin/streptomycin. Medium was replaced once a week. At DIV 14-21, microglial cells were isolated from confluent mixed glial cultures by shaking ( 80 r.p.m. for $60 \mathrm{~min}$ at $37^{\circ} \mathrm{C}$ ) and centrifuged at $600 \mathrm{~g}$ for $10 \mathrm{~min}$. The floating cells were collected, pelleted, and reseeded on 12-well plates. Microglial cells were used after another $24 \mathrm{~h}$

C $x 3 \mathrm{cr} 1^{\mathrm{CreER}}$ was induced by incubation for 5 days in medium with 4-hydroxytamoxifen (SML1666, Sigma-Aldrich, St Louis, MO, USA) diluted to a final concentration of $10 \mu \mathrm{g} / \mathrm{ml}$.

Transfections of siRNAs were performed with Viromer BLUE (TT100300, OriGene, Rockville, MD, USA) according to the manufacturer's protocol. siRNAs against cGAS (MB21D1) (SASI_Mm01_00129826, Sigma-Aldrich, St Louis, MO, USA) and STING (TMEM173) (sc-154411, Santa Cruz Biotechnology, Dallas, TX, USA) were purchased.

Tau 410 (T06-54N, Signal Chem, Biotech, Richmond, BC, Canada), Tau 410 P179A, Tau 410 P216A, Tau 410 P179A/P216A, Tau 441 (T08-54N, Signal Chem, Biotech, Richmond, BC, Canada), and Tau 441 P301S (T08-56GN, Signal Chem, Biotech, Richmond, BC, Canada) were diluted in medium to a concentration of $25 \mathrm{nM}$ and incubated for another 2 days.

Immunocytochemistry. Cultured cells were fixed with $2 \%$ paraformaldehyde (prepared in PB) at room temperature for $30 \mathrm{~min}$. After fixation, cells were treated with $0.1 \%$ Triton X-100 in PBS for 10 min, blocked with PBS containing $1 \%$ bovine serum albumin and $0.1 \%$ Triton X-100 for $30 \mathrm{~min}$ at room temperature and incubated with primary antibodies diluted in the blocking buffer. The primary antibodies were anti-PQBP1 antibody (1:250, FL-265, Santa Cruz Biotechnology, Dallas, TX, USA), anti-NFkB p65 Antibody (C-20) (1:250, sc-372, Santa Cruz Biotechnology, Dallas, TX, USA), anti-phospho-tau antibody (1:1000, AT-8, Innogenetics, Ghent, Belgium), and anti-cGAS rabbit antibody (1:500, ABF124, Merck, Darmstadt, Germany).

Luciferase assay. Primary microglia from $\mathrm{Cx} 3 \mathrm{cr}$ C $\mathrm{CreER} / \mathrm{CreER} / \mathrm{Pqbp} 1^{f l o x X / Y}$ male mouse or female: $\mathrm{Cx} 3 \mathrm{cr} 1^{\mathrm{CreER} / \mathrm{CreER} / P q b p} 1^{\text {floxX/floxX }}$ female mouse were incubated with 4-hydroxytamoxifen $(10 \mu \mathrm{g} / \mathrm{ml}$, SML1666 Sigma-Aldrich) for 5 days. Then the $\mathrm{NFkB}$ sensor primary microglia were developed by transduction with $\mathrm{NF \kappa B}$ Reporter lentivirus $\left(1 \times 10^{5} \mathrm{TU}\right)$ (CLS-013L-8, Qiagen) to $1 \times 10^{4}$ primary microglia. After 7 days of transduction, Tau 410 (T06-54N; Signal Chem, Biotech, Richmond, BC, Canada) and Tau 441 (T08-54N, Signal Chem, Biotech, Richmond, $\mathrm{BC}$, Canada) were added to the medium for $48 \mathrm{~h}$ to a final concentration of $25 \mathrm{nM}$. Luciferase assays were performed using the Dual-Glo Luciferase assay system (Promega, WI, USA).

Stereotaxic surgery and Tau injection. Mice were deeply anesthetized with isoflurane (Zoetis Japan, Tokyo, Japan) and immobilized in a stereotaxic frame. In Fig. 6, $1 \mu \mathrm{l}$ of Tau $410(2.2 \mu \mathrm{g} / \mu \mathrm{l}, 4.4 \mu \mathrm{g} / \mathrm{bilateral}$ injections, SP-502, Boston Biochem, Cambridge, MA, USA) was injected into bilateral EC at the following coordinates (from bregma): anterior/posterior (A/P): $-4.7 \mathrm{~mm}$, medial/lateral $(\mathrm{M} / \mathrm{L}): \pm 3.3 \mathrm{~mm}$, dorsal/ventral (D/V): $-2.0 \mathrm{~mm}$ from brain surface. In Figs. 7, 9, 10 and Supplementary Fig. 9, non-polymerized and polymerized Tau 410, Tau 410 P179A, Tau 410 P216A, Tau 410 P179A/P216A, Tau 441, and Tau 441 P301S were labeled with Alexa Fluor 488 Microscale Protein Labeling Kit (A30006, Thermo Fisher Scientific, Waltham, MA, USA), and $4 \mu \mathrm{l}$ of each labeled tau $(0.5 \mu \mathrm{g} / \mu \mathrm{l}, 4 \mu \mathrm{g} / \mathrm{bilateral}$ injections) were injected at a rate of $0.2 \mu \mathrm{l} / \mathrm{min}$ via a glass micropipette. To avoid any suction effect of the solution injected, micropipettes were left in place for an additional $5 \mathrm{~min}$ before being slowly withdrawed. Afterwards, the incision was closed by suture.

Mouse behavioral analysis. Spatial memory during exploratory behavior was assessed by the Y-maze test, using Y-shape maze with three identical arms interconnected at an angle of $120^{\circ}$ (YM-3002, O'HARA \& Co., Ltd, Tokyo, Japan). After mice were habituated to the procedure room at least $30 \mathrm{~min}$ prior to testing, they were put at the end of one arm and allowed to explore freely through the maze during an $8 \mathrm{~min}$ session. The percentage of spontaneous alterations (indicated as an alteration score) was calculated based on the sequence of arm entries, dividing the number of entries into a new arm different from the previous one by the total number of transfers from one arm to another.

In the Morris water maze test, mice were trained to escape from water by swimming onto a hidden platform. Water temperature was maintained at $21 \pm 1{ }^{\circ} \mathrm{C}$. Mice performed four training trials per day $(60 \mathrm{~s})$ for 4 days, and the latency to reach the hidden platform was recorded.

Immunohistochemistry. Mouse brains injected with non-fluorescently labeled tau protein were fixed in $4 \%$ paraformaldehyde for $12 \mathrm{~h}$ and embedded in paraffin. Sagittal or coronal sections (thickness, $5 \mu \mathrm{m}$ ) were de-paraffinized in xylene, rehydrated, dipped in $0.01 \mathrm{M}$ citrate buffer (pH 6.0), and microwaved at $120^{\circ} \mathrm{C}$ for $15 \mathrm{~min}$. Mice injected with fluorescently labeled tau protein were perfused with $0.9 \% \mathrm{NaCl}$ prior to brain tissue fixation, followed by tissue processing to form 25 $\mu \mathrm{m}$-thick frozen sections. Sections were blocked with 10\% FBS containing PBS. Immunohistochemistry was performed using the following primary antibodies: mouse anti-PQBP1 (1:200, sc-374260, Santa Cruz Biotechnology, Dallas, TX, USA); rabbit anti-PQBP1 (1:200, A302-801A, Bethyl Laboratory, Montgomery, TX, USA) rabbit anti-Ibal (1:1000, 019-19741, Wako, Osaka, Japan); goat anti-Iba1 (1:500, 011-27991, Wako, Osaka, Japan); goat anti-Ibal (1:500, ab107159, Abcam, Cambridge, UK); rabbit anti-NFkB p65 (C-20) (1:250, sc-372, Santa Cruz Biotechnology, Dallas, TX, USA); mouse anti-phospho-tau (1:1000, AT-8, Innogenetics, Ghent, Belgium); mouse anti-tau (1:500, MA5-15108, Thermo Fisher Scientific, Waltham, MA, USA); rabbit anti-cGAS (1:500, ABF124, Merck, Darmstadt, Germany); mouse anti-LPL (1:100, ab21356, Abcam, Cambridge, UK); rabbit antiMAP2 (1:1000, ab32454, Abcam, Cambridge, UK); mouse anti-GFAP-Cy3 (1:5000, C9205, Sigma-Aldrich, St. Louis, MO, USA); mouse anti-NeuN (1:1000, ab104224, Abcam, Cambridge, UK). Secondary antibodies were as follows: donkey anti-goat IgG Alexa568 (1:1000, A11057, Molecular Probes, Eugene, OR, USA); donkey antimouse IgG Alexa488 (1:1000, A21202, Molecular Probes, Eugene, OR, USA); donkey anti-rabbit IgG Alexa647 (1:1000, A31573, Molecular Probes, Eugene, OR, USA). Nuclei were stained with DAPI $(0.2 \mu \mathrm{g} / \mathrm{ml}$ in PBS, D523, DOJINDO Laboratories, Kumamoto, Japan).

For nick end labeling, frozen sections were washed three times with PBS containing $0.1 \%$ Tween-20 (PBST) at room temperature. The sections were incubated with labeling reaction mix [Biotin-16-dUTP (11093070910, Roche, Mannheim, Germany) and terminal transferase (03333574001, Roche, Mannheim, Germany)] at $37^{\circ} \mathrm{C}$ for $2 \mathrm{~h}$, washed with PBST three times, and then incubated with Alexa Fluor 633-conjugated streptavidin (S21375, Thermo Fisher Scientific) at room temperature for $1 \mathrm{~h}$.

Western blot analysis. Mouse primary microglia in culture dishes were washed three times with PBS and dissolved in sample buffer containing $62.5 \mathrm{mM}$ Tris- $\mathrm{HCl}$, $\mathrm{pH} 6.8,2 \%(\mathrm{w} / \mathrm{v})$ SDS, $2.5 \%(\mathrm{v} / \mathrm{v}) 2$-mercaptoethanol, $10 \%(\mathrm{v} / \mathrm{v})$ glycerol, and $0.0025 \%(\mathrm{w} / \mathrm{v})$ bromophenol blue. Samples were separated by SDS-PAGE, transferred to Immobilon-P polyvinylidene difluoride membranes (Millipore, Burlington, MA, USA) using the semi-dry method, blocked with $5 \%$ milk in TBST (10 mM Tris- $\mathrm{HCl} \mathrm{pH} 8.0,150 \mathrm{mM} \mathrm{NaCl}$, and $0.05 \%$ Tween-20), and reacted with the following primary and secondary antibodies diluted in Can Get Signal solution 
a

\begin{tabular}{|c|c|c|c|c|}
\hline \multicolumn{2}{|c|}{ Y-maze test } & \multicolumn{3}{|c|}{ Morris water maze test } \\
\hline $\begin{array}{ll}\text { PBS } & \text { O Tau410 } \\
& \text { О Tau410 P179A }\end{array}$ & $\begin{array}{l}\text { Tau410 P216A } \\
\text { Tau410 P179A / P216A }\end{array}$ & $\begin{array}{l}\text { OTau441 } \\
\text { Tau441 P301S }\end{array}$ & $\begin{array}{l}\# \#: P<0.001 \\
\# \#: P<0.01\end{array}$ & $\begin{array}{r}\text { \#: } P<0.05 \text { by Dunnett's test } \\
(N=6)\end{array}$ \\
\hline \multicolumn{2}{|c|}{ Non-polymerized } & \multicolumn{3}{|c|}{ Non-polymerized } \\
\hline Non-induced Pqbp1-cKO & Induced Pqbp1-cKO & Non-induc & Pqbp1-cKO & Induced Pqbp1-cKO \\
\hline
\end{tabular}
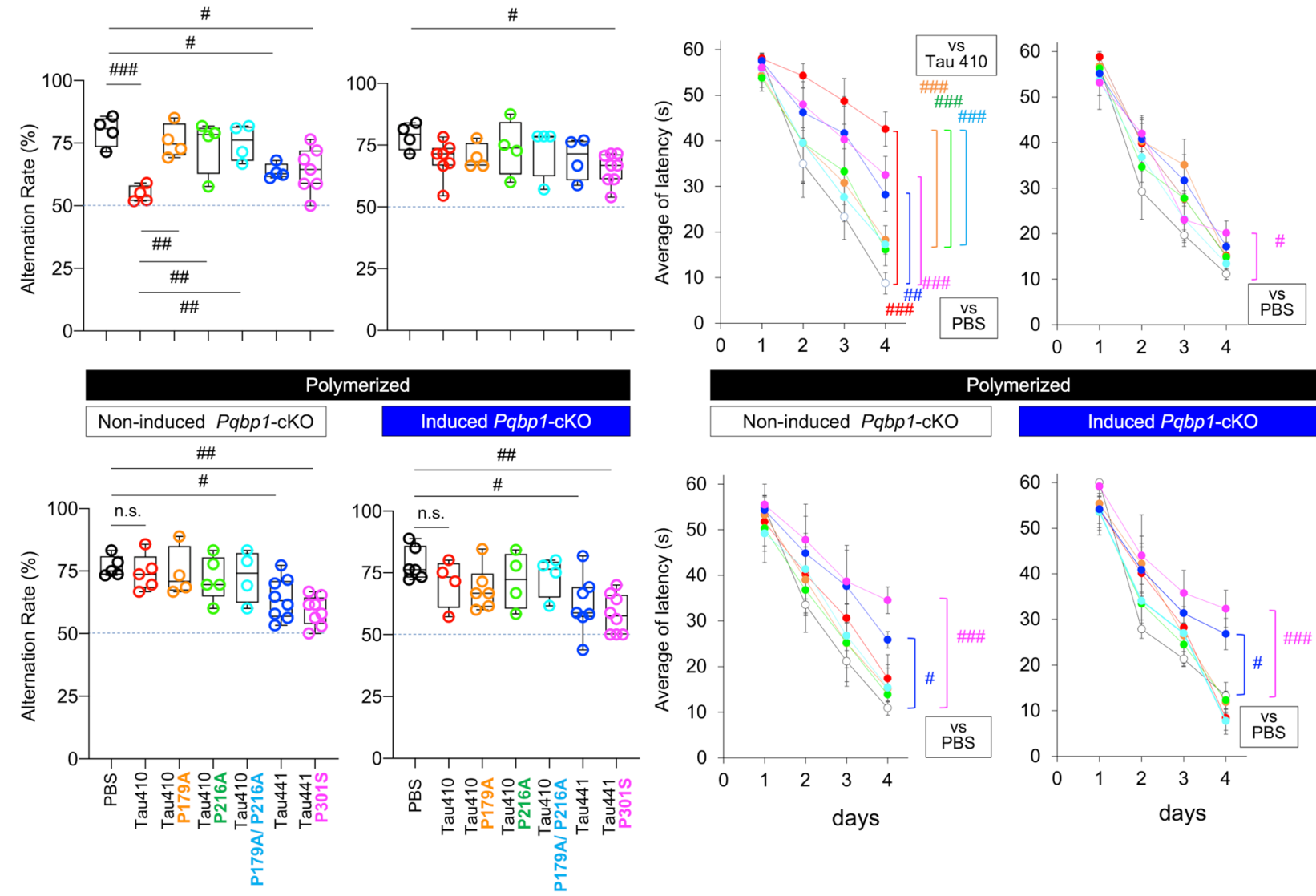

\section{Polymerized}

Induced Pqbp1-cKO
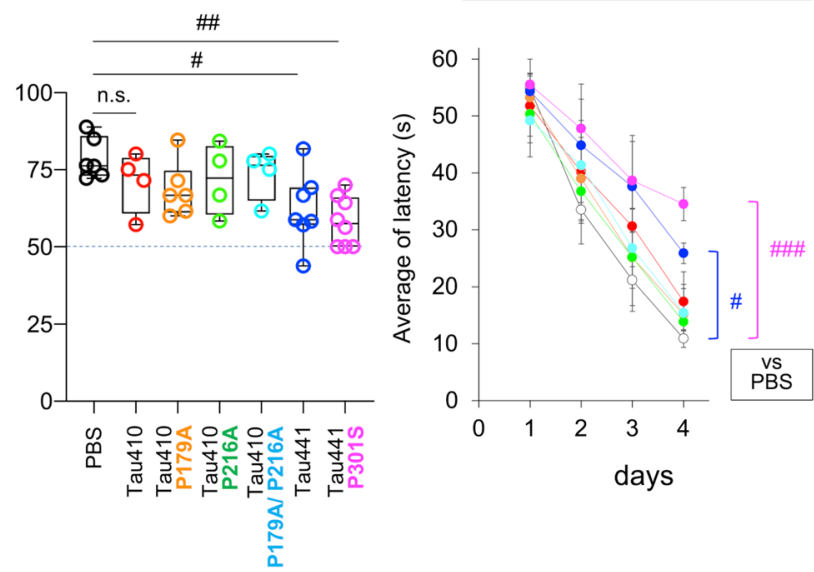

Polymerized

Non-induced Pqbp1-cKO

Induced Pqbp1-cKO

b
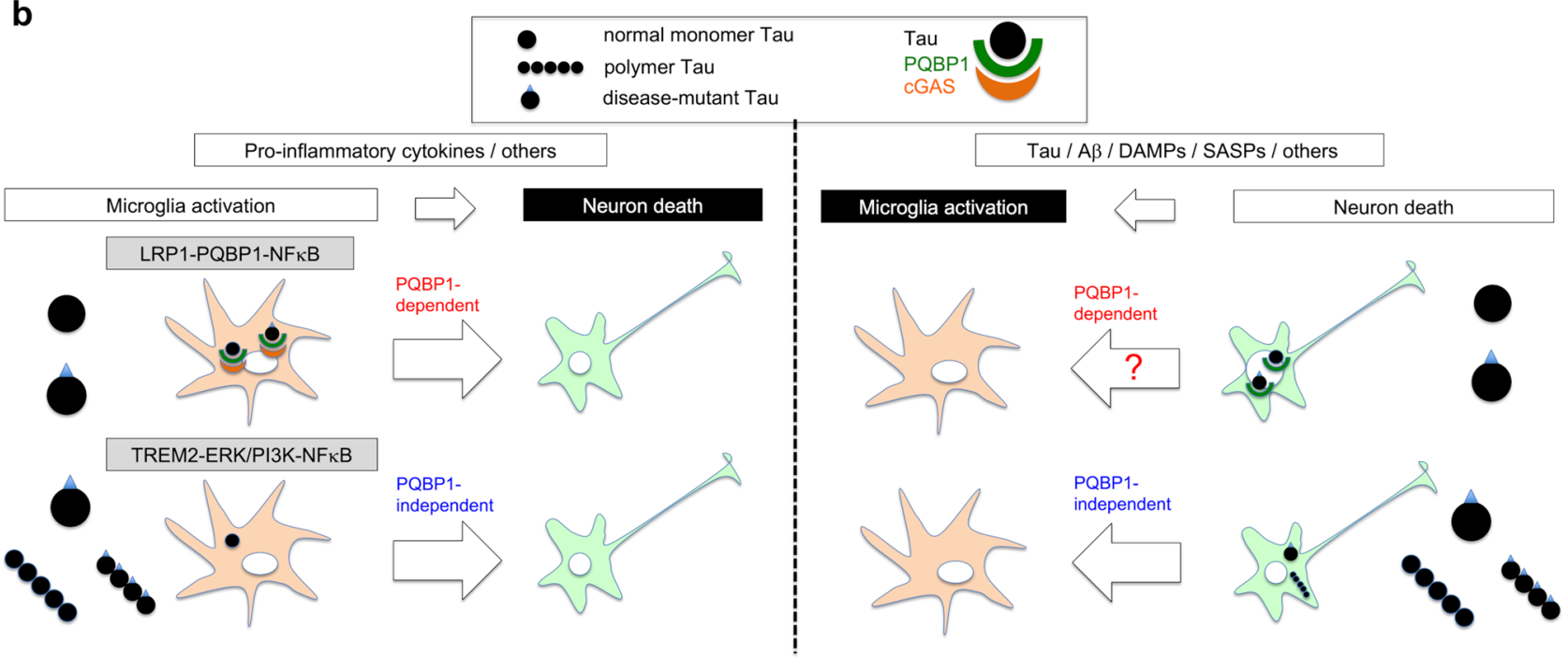

Fig. 10 PQBP1 depletion in microglia recovers memory functions impaired by Tau. a Memory dysfunction after injection of various species of tau in nonpolymerized or polymerized state was evaluated by $Y$-maze test and Morris water maze test. Protocol from injection to memory function tests in various conditions is shown in Fig. 6a. Y-maze test: $N$ number in each group is indicated in Source data. In Morris water maze test, values in each group are presented as mean $\pm \mathrm{SEM} ; N=6$ mice/group. $\# P<0.05, \# \# P<0.01, \# \# P<0.001$ in Dunnett's test. Box plots show the median, quartiles. and whiskers that represent data outside the 25th to 75th percentile range. $\mathbf{b}$ PQBP1-depndent and PQBP1-independent pathways mediating microglia activation to neuronal cell death or neuronal cell death to microglia activation. Different types of tau species trigger distinct pathways. 
(Toyobo, Osaka, Japan as follows: mouse anti-tau (1:3000, MAB361, Millipore, Burlington, MA, USA); rabbit anti-PQBP1 (1:500, sc-32910, Santa Cruz Biotechnology, Dallas, TX, USA); rabbit anti-cGAS (1:1000, ABF124, Merck, Darmstadt, Germany); rabbit anti-STING (1:3000, 13647S, Cell Signaling Technology, Danvers, MA, USA); rabbit anti-phospho-Ser536-NFkB (1:1000, 3033S, Cell Signaling Technology, Danvers, MA, USA); rabbit anti-phospho-Ser396-IRF3 (1:1000, 4947S, Cell Signaling Technology, Danvers, MA, USA); mouse anti-GAPDH (1:5000, MAB374, Millipore, Burlington, MA, USA); mouse anti-tau (1:10,000 ab80579, Abcam, Cambridge, UK); rabbit anti-PQBP1 (1:1000, A302-801A, Bethyl, Montgomery, TX, USA); HRP-conjugated anti-mouse IgG (1:3000, NA931VA, GE Healthcare, Chicago, IL, USA); and HRP-conjugated anti-rabbit IgG (1:3000, NA934VS, GE Healthcare, Chicago, IL, USA). ECL Select Western Blotting Detection Reagent (RPN2235, Cytiva, Marlborough, MA, USA) and an ImageQuant LAS 500 luminescent image analyzer were used to detect proteins. Full-scan images are displayed in Source data file.

Quantitative RT-PCR. Total RNA was isolated from primary microglia at 2 days after heparin and Tau 410/441 (polymerized and not polymerized) incubation with NucleoSpin RNA XS (U0902A, TaKaRa, Shiga, Japan). Total RNA was isolated from entorhinal cortex tissues of 14 groups of Pqbp1-cKO mice at 21 days after tau proteins/PBS injection with RNeasy mini kit (74106, Qiagen, Limburg, The Netherlands). To eliminate genomic DNA contamination, on-column digestion of DNA was carried out with DNase I. Reverse transcription was performed using the SuperScript VILO cDNA Synthesis kit (11754-250, Invitrogen, Carlsbad, CA, USA). Quantitative PCR analyses were performed with the 7500 Real-Time PCR System (Applied Biosystems, Foster City, CA, USA) using the Thunderbird SYBR Green (QPS-201, TOYOBO, Osaka, Japan) and assessed by the standard curve method. The primer sequences were:

mouse $\operatorname{Tnf}$, forward primer: $5^{\prime}$-TGCTTGTTGACAGCGGTCC- $3^{\prime}$ and reverse primer: 5'-ACTGGCCATCGTGGAGGTAC- $3^{\prime}$

mouse Isg54, forward primer: $5^{\prime}$-AGCAAGATGCACCAAGATGA-3' and reverse primer: $5^{\prime}$-CTGTGTCAAAGCGCTCAAAG- ${ }^{\prime}$

mouse Ifn $\beta$, forward primer: $5^{\prime}$-GCCTTTGCCATCCAAGAGATGC- $3^{\prime}$ and reverse primer: $5^{\prime}$-ACACTGTCTGCTGGTGGAGTTC- ${ }^{\prime}$

mouse Cxcl10, forward primer: $5^{\prime}$-ATCATCCCTGCGAGCCTATCCT- $3^{\prime}$ and reverse primer: $5^{\prime}$-GACCTTTTTTGGCTAAACGCTTTC-3'

mouse Gapdh, forward primer: $5^{\prime}$-TGAACGGGAAGCTCACTGG- ${ }^{\prime}$ and reverse primer: $5^{\prime}$-TCCACCACCCTGTTGCTGTA- $3^{\prime}$

The PCR conditions for amplification were $95^{\circ} \mathrm{C}$ for $10 \mathrm{~min}$ for enzyme activation, $95^{\circ} \mathrm{C}$ for $15 \mathrm{~s}$ for denaturation, and $60^{\circ} \mathrm{C}$ for $1 \mathrm{~min}$ for extension $(40$ cycles). The expression levels of individual genes were normalized against the expression of GAPDH and calculated as a relative expression level.

\section{Live imaging of microglia in primary culture. Mouse primary microglia were} prepared from Pqbp1-cKO or C57BL/6 mice at P3. At DIV 14-21, microglial cells were isolated from confluent mixed glial cultures and reseeded on eight-well chambers. After $24 \mathrm{~h}$, pEGFP-N1-PQBP1 was transfected into microglia using Viromer RED (TT100302, OriGene, Rockville, MD, USA) for $24 \mathrm{~h}$. Time-lapse image was acquired with an Olympus FV10i-W laser-scanning microscope at $\times 60$ magnification (Olympus, Tokyo, Japan) every $15 \mathrm{~min}$ for the next $42 \mathrm{~h}$. TAMRAlabeled Tau proteins were added to the medium at $18 \mathrm{~h}$ post-transfection. The chamber was kept at $37^{\circ} \mathrm{C}$ with $5 \% \mathrm{CO}_{2}$.

Live imaging of microglia in in vivo mouse brains. Before siRNA injection, siRNA was labeled with Label IT siRNA Tracker Cy5 Kit without Transfection Reagent (MIR7213, Mirus, WI, USA) according to the manufacturer's procedures. Under anesthesia with $1 \%$ isoflurane, 300 ng Cy5-labeled-siRNA (mouse PQBP1siRNA, sc-38200, Santa Cruz Biotechnology, Dallas, TX, USA) in $1 \mu \mathrm{l}$ volume was injected into the retrosplenial cortex (anteroposterior, $-3.0 \mathrm{~mm}$ form bregma; lateral, $0.6 \mathrm{~mm}$; depth, $0.5 \mathrm{~mm}$ ) of $C x 3 c r 1-G F P$ mouse (005582, Jackson Laboratory, Bar Harbor, MW, USA) at 21 weeks of age using in vivo jetPEI (201-10G, Polyplus-transfection, Illkirch, France). After $16 \mathrm{~h}$, the skull was thinned with a high-speed micro-drill in the mouse splenial cortex. Then, the head of each mouse was immobilized by a head plate on a custom machine stage mounted on the microscope table. Two-photon imaging was performed at $5 \mathrm{~min}$ intervals for 30 min using a laser-scanning microscope system FV1000MPE2 (Olympus, Tokyo, Japan) equipped with an upright microscope (BX61WI, Olympus, Japan), a waterimmersion objective lens (XLPlanN25xW; numerical aperture, 1.05), and a pulsed laser (MaiTaiHP DeepSee, Spectra Physics, Santa Clara, CA, USA). Then, after injecting $2.2 \mu \mathrm{g}$ TAMRA-labeled Tau 410 (anteroposterior, $-1.0 \mathrm{~mm}$ form bregma; lateral, $0.6 \mathrm{~mm}$; depth, $0.5 \mathrm{~mm}$ ), two-photon imaging was performed again at $5 \mathrm{~min}$ intervals for $30 \mathrm{~min}$.

Live imaging of neuron in primary culture. Mouse primary cortical neurons were prepared from E17 C57BL/6J mouse embryos. Cerebral cortices were dissected and incubated with $0.05 \%$ trypsin in PBS at $37^{\circ} \mathrm{C}$ for $15 \mathrm{~min}$ and dissociated by pipetting. The cells were passed through a $70 \mu \mathrm{m}$ cell strainer (22-363-548, Thermo Fisher Scientific, MA, USA), collected by centrifugation, and cultured in neurobasal medium (21103049, Thermo Fisher Scientific, Waltham, MA, USA) containing 2\% B27 (17504044, Thermo Fisher Scientific, Waltham, MA, USA), $0.5 \mathrm{mM}$ L-glutamine, and $1 \%$ penicillin/streptomycin (15140-122, Thermo Fisher Scientific, Waltham, MA, USA). Five days later, time-lapse images of neurons were acquired at $\times 60$ magnification on an Olympus FV10i-W laser-scanning microscope (Olympus, Tokyo, Japan) at $1 \mathrm{~h}$ intervals for $24 \mathrm{~h}$. The chamber was kept at $37^{\circ} \mathrm{C}$ with $5 \% \mathrm{CO}_{2}$. The ratio of cell death patterns was counted $18 \mathrm{~h}$ after addition of labeled tau.

Knockout of LRP1 and TREM2 in primary microglia. In total, $0.6 \mu \mathrm{g}$ of Control Double Nickase Plasmid (sc-437281, Santa Cruz Biotechnology, Dallas, TX, USA) LRP1 Double Nickase Plasmid (sc-421464-NIC, Santa Cruz Biotechnology, Dallas, TX, USA) and TREM2 Double Nickase Plasmid (sc-429903-NIC, Santa Cruz Biotechnology, Dallas, TX, USA) were electroporated into suspended microglia using 4D-Nucleofector (pulse program: CV-110) (4D-Nucleofector Core Unit, \#AAF-1002B, LONZA, NJ, USA). The electroporated cells were cultured on LabTek II chambered coverglass.

To test knockout efficiency, cells were fixed in $0.4 \%$ FA, blocked with $10 \%$ FBS in PBS for $60 \mathrm{~min}$ at RT, incubated with primary antibody for $60 \mathrm{~min}$, and with secondary antibodies for $60 \mathrm{~min}$ at RT. The antibodies used for immunocytochemistry were diluted as follows: mouse anti-TREM2 (1:100, sc-373828, Santa Cruz Biotechnology, Dallas, TX, USA); rabbit anti-PQBP1 (1:150, Bethyl Laboratories, A302-801A, Montgomery,TX, USA); Cy5-conjugated anti-mouse IgG (1:500, 715-175-151, Jackson Laboratory, Bar Harbor, ME, USA); and Alexa Fluor 405-conjugated anti-rabbit IgG (1:1000, A48258, Molecular Probes, Eugene, OR, USA). For multiple co-staining, mouse anti-LRP1 antibody (1:250, sc-57353, Santa Cruz Biotechnology, Dallas, TX, USA) was labeled by Zenon Secondary Detection-Based Antibody Labeling Kits (Zenon Alexa Fluor 555 Rabbit IgG Labeling Kit, Z-25305, Thermo Fisher Scientific, Waltham, MA, USA).

To monitor NFkB activity, NFkB-RFP lentivirus (LVP966-P, GenTarget Inc., San Diego, CA, USA) driven by a minimal CMV promoter and NFkB transcriptional response element (NFkB-TRE) was infected to cells $\left(1 \times 10^{4}\right.$ cells/ well) at MOI of 5 . At $72 \mathrm{~h}$ after infection, Tau 410 (T06-54N, Signal Chem, Biotech, Richmond, BC, Canada) labeled with Alexa Fluor 555 Microscale Protein Labeling Kit (A30007, Thermo Fisher Scientific, Waltham, MA, USA) was added to microglia at $25 \mathrm{nM}$ and incubated for another 2 days. The fluorescent signal was analyzed by confocal microscopy (FV1200IXGP44, Olympus, Tokyo, Japan). The luciferase assay was also used to monitor NFkB activity similarly to the abovementioned method.

Infection of AAV-PQBP1. Mouse primary cortical neurons were prepared from E17 C57BL/6J mouse embryos. Cerebral cortices were dissected, and incubated with $0.05 \%$ trypsin in PBS at $37^{\circ} \mathrm{C}$ for $15 \mathrm{~min}$ and dissociated by pipetting. The cells were passed through a 70- $\mu \mathrm{m}$ cell strainer (22-363-548, Thermo Fisher Scientific, MA, USA), collected by centrifugation, and cultured in neurobasal medium (21103049, Thermo Fisher Scientific, Waltham, MA, USA) containing 2\% B27 (17504044, Thermo Fisher Scientific, Waltham, MA, USA), $0.5 \mathrm{mM}$ L-glutamine, and $1 \%$ penicillin/streptomycin (15140-122, Thermo Fisher Scientific, Waltham, MA, USA). Forty-eight hours later, the medium was changed to that containing $0.5 \mu \mathrm{M}$ AraC (C3631, Sigma-Aldrich, St. Louis, MO, USA). Mouse primary microglia were prepared from C57BL/6 mice at P3 as described above. Primary microglia and primary neuron were infected by AAV1-CMV-hPQBP1 (ref. ${ }^{35}$ ) (MOI: 5000) in the culture medium for 5 days. For western blot, after culture medium was removed and the cells were washed with PBS, cells were added to lysis buffer (10 mM Tris- $\mathrm{HCl}, \mathrm{pH} 7.5,10 \mathrm{mM} \mathrm{NaCl}, 0.5 \mathrm{mM}$ EDTA, $1 \%$ NP40, $0.5 \%$ protease inhibitor cocktail (539134, Calbiochem, San Diego, CA, USA)). Lysates were rotated for $60 \mathrm{~min}$ at $4^{\circ} \mathrm{C}$, and then centrifuged at $12,000 \times g$ for $20 \mathrm{~min}$ at $4^{\circ} \mathrm{C}$. After adding $1 \% \beta$-mercaptoethanol to the supernatants, the supernatants were rotated for $5 \mathrm{~min}$ at $4{ }^{\circ} \mathrm{C}$, an equal volume of sample buffer $(50 \mathrm{mM}$ Tris- $\mathrm{HCl}$, $\mathrm{pH} 6.5,2 \%$ SDS, $10 \%$ glycerol, $0.005 \% \mathrm{BPB}$ ) was added, and the samples were boiled at $100{ }^{\circ} \mathrm{C}$ for $3 \mathrm{~min}$ before SDS-PAGE.

Chemotaxis assay. Chemotaxis assay of mouse primary microglia with and without PQBP1 depletion was performed with Boyden chambers (CBA-105, CytoSelectTM 96-Well Cell Migration Assay, Cell Biolabs, San Diego, CA, USA) following the manufacturer's protocol ${ }^{85}$. Overnight starvation was performed prior to running the assay. Cell suspensions of microglia incubated with and without tamoxifen were seeded into the upper membrane chambers at $2.5 \times 10^{4} \mathrm{cells} /$ well in $100 \mu \mathrm{l}$ serum free media. Media (150 $\mu \mathrm{l})$ containing $10 \%$ FBS was added to the lower chambers. Then, cells were allowed to migrate for $4 \mathrm{~h}$ at $37^{\circ} \mathrm{C}$ in an incubator enriched with $5 \% \mathrm{CO}_{2}$. At the end of the assay, migrated cells, which clung to the bottom side of the membrane, were dissociated by adding cell detachment buffer. Then, cells were lysed and quantified using CyQuant GR Fluorescent Dye by a FLUOstar OPTIMA-6 microplate reader (BMG Labtech, Durham, NC, USA) using $480 \mathrm{~nm}$ excitation and $520 \mathrm{~nm}$ emission wavelengths.

Phagocytosis assay. Phagocytic activity of mouse primary microglia with and without PQBP1 depletion was performed with the Phagocytosis Assay Kit (500290, Cayman Chemical, Ann Arbor, MI, USA) according to the manufacturer's 
protocol ${ }^{86}$. Cells were seeded into a 96-well plate at $1 \times 10^{4}$ cells $/$ well. After the induction of PQBP1 depletion as previously described, latex beads coated with rabbit IgG-FITC conjugates were diluted by culture medium at a final concentration of $1: 100$. Then cells were incubated with $200 \mu$ l media containing latex beads for $4 \mathrm{~h}$ followed by a 1-min incubation with trypan blue to quench nonphagocytosed bead fluorescence. The fluorescence intensity was measured by a FLUOstar OPTIMA-6 microplate reader (BMG Labtech, Durham, NC, USA) at $485 \mathrm{~nm}$ excitation and $535 \mathrm{~nm}$ emission wavelengths. Cells were then fixed and counterstained with anti-Ibal and -PQBP1 antibodies.

Statistics. Statistical analyses for biological experiments were performed using Graphpad Prism 8, R version 3.6.2., or Microsoft Excel for Microsoft 365.

Biological data following a normal distribution are presented as the mean \pm SEM. Tukey's HSD test was applied. The significance level was set at 1 or $5 \%$.

A box plot was used to depict distribution of observed data, and the data are also plotted as dots. Box plots show medians, quartiles, and whiskers, which represent data outside the 25 th-75th percentile range.

For Fig. 9b, a three-way ANOVA was used to examine mutual effects among the examination conditions (tau mutation, polymerization, Pqbp1-cKO) on neuronal cell death. No interaction was detected between tau mutation and polymerization $(P=0.1336)$, while interaction was detected between $P q b p 1$-cKO and polymerization $(P=0.0022)$ or between $P q b p 1$-cKO and mutation $(P=0.0408)$. Therefore, a post hoc Tukey's HSD test was used to compare the number of dead cells in four types of non-polymerized PQBP1-binding mutations of tau. Significant difference was observed between Tau 410 and Tau 410 P179A $(P=0.0144)$, Tau 410 P216A $(P=0.0028)$, or Tau 410 P179A/P216A $(P=0.0078)$. For each type of tau, a post hoc Tukey's HSD test was used to compare the cell death numbers among all cases irrespective of polymerized/non-polymerized or induced/noninduced Pqbp1-cKO conditions. A significant difference was observed in Tau 410 (non-polymerized/non-induced vs non-polymerized/induced, $P=0.0001$ ), but no significance was found in Tau 410 P179A, Tau 410 P216A, or Tau 410 P179A/ P216A.

Ethics for animal experiments. All animal experiments were strictly performed according to the ARRIVE guidelines (Animal Research: Reporting in vivo Experiments) for the Care and Use of Laboratory Animals of the National Institutes of Health. The experiments were approved by the Committees on Gene Recombination Experiments and Animal Experiments of Tokyo Medical and Dental University (G2018-082C and A2019-218C2).

Ethics for human experiments. All experiments using human samples were conducted with informed consent and in accordance with the approved guidelines for human experimental research. All the experiments were approved by the Committee on Human Ethics of the Tokyo Medical and Dental University (O2014005-13/O2020-002).

Reporting summary. Further information on research design is available in the Nature Research Reporting Summary linked to this article.

\section{Data availability}

All relevant data are available within the article and its Supplementary Information files. UniProt database of subcellular locations was used for STING (https://www.uniprot.org/ uniprot/Q86WV6) and for cGAS (https://www.uniprot.org/uniprot/Q8N884). Human FUS, TDP43, hnRNPA1, and hnRNPA2B1 protein sequences were retrieved from UniProt (https://www.uniprot.org/). Source data are provided with this paper.

Received: 1 December 2020; Accepted: 23 October 2021;

Published online: 15 November 2021

\section{References}

1. Wyss-Coray, T. \& Mucke, L. Inflammation in neurodegenerative disease-a double-edged sword. Neuron 35, 419-432 (2002).

2. Selkoe, D. J. Alzheimer's disease: genes, proteins, and therapy. Physiol. Rev. 81, 741-766 (2001).

3. Scaffidi, P., Misteli, T. \& Bianchi, M. E. Release of chromatin protein HMGB1 by necrotic cells triggers inflammation. Nature 418, 191-195 (2002).

4. Fujita, K. et al. HMGB1, a pathogenic molecule that induces neurite degeneration via TLR4-MARCKS, is a potential therapeutic target for Alzheimer's disease. Sci. Rep. 6, 31895 (2016).

5. Glass, C. K., Saijo, K., Winner, B., Marchetto, M. C. \& Gage, F. H. Mechanisms underlying inflammation in neurodegeneration. Cell 140, 918-934 (2010).

6. Lemke, G. \& Rothlin, C. V. Immunobiology of the TAM receptors. Nat. Rev. Immunol. 8, 327-336 (2008).
7. Schetters, S. T. T., Gomez-Nicola, D., Garcia-Vallejo, J. J. \& Van Kooyk, Y. Neuroinflammation: microglia and $\mathrm{T}$ cells get ready to tango. Front. Immunol. 8, 1905 (2018).

8. Yoh, S. M. et al. PQBP1 is a proximal sensor of the cGAS-dependent innate response to HIV-1. Cell 161, 1293-1305 (2015).

9. Lian, H. et al. ZCCHC3 is a co-sensor of cGAS for dsDNA recognition in innate immune response. Nat. Commun. 9, 3349 (2018).

10. Liu, Z. S. et al. G3BP1 promotes DNA binding and activation of cGAS. Nat. Immunol. 20, 18-28 (2019).

11. Imafuku, I. et al. Polar amino acid-rich sequences bind to polyglutamine tracts. Biochem. Biophys. Res. Commun. 253, 16-20 (1998).

12. Waragai, $M$. et al. PQBP-1, a novel polyglutamine tract-binding protein, inhibits transcription activation by Brn-2 and affects cell survival. Hum. Mol. Genet. 8, 977-987 (1999).

13. Okazawa, H. et al. Interaction between mutant ataxin-1 and PQBP-1 affects transcription and cell death. Neuron 34, 701-713 (2002).

14. Okazawa, H., Sudol, M. \& Rich, T. PQBP-1 (Np/PQ): a polyglutamine tractbinding and nuclear inclusion-forming protein. Brain Res. Bull. 56, 273-280 (2001).

15. Sudol, M., McDonald, C. B. \& Farooq, A. Molecular insights into the WW domain of the Golabi-Ito-Hall syndrome protein PQBP1. FEBS Lett. 586, 2795-2799 (2012).

16. Tapia, V. E. et al. Y65C missense mutation in the WW domain of the GolabiIto-Hall syndrome protein PQBP1 affects its binding activity and deregulates pre-mRNA splicing. J. Biol. Chem. 285, 19391-19401 (2010).

17. Pucheta-Martinez, E. et al. Changes in the folding landscape of the WW domain provide a molecular mechanism for an inherited genetic syndrome. Sci. Rep. 6, 30293 (2016).

18. Takahashi, M. et al. Polyglutamine tract binding protein-1 is an intrinsically unstructured protein. Biochim. Biophys. Acta-Proteins Proteomics 1794, 936-943 (2009)

19. Rees, M. et al. Solution model of the intrinsically disordered polyglutamine tract-binding protein-1. Biophys. J. 102, 1608-1616 (2012).

20. Mizuguchi, M. et al. Mutations in the PQBP1 gene prevent its interaction with the spliceosomal protein U5-15kD. Nat. Commun. 5, 3822 (2014).

21. Mizuguchi, M. et al. Allosteric modulation of the binding affinity between PQBP1 and the spliceosomal protein U5-15kD. FEBS Lett. 590, 2221-2231 (2016).

22. Rahman, S. K., Okazawa, H. \& Chen, Y. W. Frameshift PQBP-1 mutants K192Sfs * 7 and R153Sfs * 41 implicated in X-linked intellectual disability form stable dimers. J. Struct. Biol. 206, 305-313 (2019).

23. Kalscheuer, V. M. et al. Mutations in the polyglutamine binding protein 1 gene cause X-linked mental retardation. Nat. Genet. 35, 313-315 (2003).

24. Claus, L. et al. Novel truncating mutations in the Polyglutamine Tract Binding Protein 1 Gene (PQBP1) cause renpenning syndrome and X-linked mental retardation in another family with microcephaly. Am. J. Hum. Genet. 74, 777-780 (2004).

25. Stevenson, R. E. et al. Renpenning syndrome comes into focus. Am. J. Med. Genet. 134 A, 415-421 (2005).

26. Lubs, H. et al. Golabi-Ito-Hall syndrome results from a missense mutation in the WW domain of the PQBP1 gene. J. Med. Genet. 43, e30 (2006).

27. Waragai, M. et al. PQBP-1/Npw38, a nuclear protein binding to the polyglutamine tract, interacts with $\mathrm{U} 5-15 \mathrm{kD} / \mathrm{dim} 1 \mathrm{p}$ via the carboxyl-terminal domain. Biochem. Biophys. Res. Commun. 273, 592-595 (2000).

28. Zhang, Y. Z. et al. Evidence that Dim1 associates with proteins involved in pre-mRNA splicing, and delineation of residues essential for Dim1 interactions with hnRNP F and Npw38/PQBP-1. Gene 257, 33-43 (2000).

29. Llorian, M., Beullens, M., Andrés, I., Ortiz, J. M. \& Bollen, M. SIPP1, a novel pre-mRNa splicing factor and interactor of protein phosphatase-1. Biochem. J. 378, 229-238 (2004).

30. Morchikh, M. et al. HEXIM1 and NEAT1 long non-coding RNA form a multi-subunit complex that regulates DNA-mediated innate immune response. Mol. Cell 67, 387-399.e5 (2017).

31. Iwasaki, Y. \& Thomsen, G. H. The splicing factor PQBP1 regulates mesodermal and neural development through FGF signaling. Dev 141, 3740-3751 (2014).

32. Ikeuchi, Y. et al. The XLID protein PQBP1 and the GTPase Dynamin 2 define a signaling link that orchestrates ciliary morphogenesis in postmitotic neurons. Cell Rep. 4, 879-889 (2013).

33. Wang, Q., Moore, M. J., Adelmant, G., Marto, J. A. \& Silver, P. A. PQBP1, a factor linked to intellectual disability, affects alternative splicing associated with neurite outgrowth. Genes Dev. 27, 615-626 (2013).

34. Ito, H. et al. In utero gene therapy rescues microcephaly caused by Pqbp1hypofunction in neural stem progenitor cells. Mol. Psychiatry 20, 459-471 (2015).

35. Tanaka, $\mathrm{H}$. et al. The intellectual disability gene PQBP1 rescues Alzheimer's disease pathology. Mol. Psychiatry 23, 2090-2110 (2018). 
36. Talukdar, H. A. et al. Cross-tissue regulatory gene networks in coronary artery disease. Cell Syst. 2, 196-208 (2016).

37. Okazawa, H. PQBP1, an intrinsically disordered/denatured protein at the crossroad of intellectual disability and neurodegenerative diseases. Neurochem. Int. 119, 17-25 (2018).

38. Nussbaum, J. M. et al. Prion-like behaviour and tau-dependent cytotoxicity of pyroglutamylated amyloid- $\beta$. Nature 485, 651-655 (2012).

39. Lasagna-Reeves, C. A. et al. Alzheimer brain-derived tau oligomers propagate pathology from endogenous tau. Sci. Rep. 2, 700 (2012).

40. Sanders, D. W. et al. Distinct tau prion strains propagate in cells and mice and define different tauopathies. Neuron 82, 1271-1288 (2014).

41. Busch, A. et al. Mutant huntingtin promotes the fibrillogenesis of wild-type huntingtin: a potential mechanism for loss of huntingtin function in Huntington's disease. J. Biol. Chem. 278, 41452-41461 (2003).

42. Fernández-Nogales, M. et al. Huntington's disease is a four-repeat tauopathy with tau nuclear rods. Nat. Med. 20, 881-885 (2014).

43. Raiborg, C., Wenzel, E. M. \& Stenmark, H. ER-endosome contact sites: molecular compositions and functions. EMBO J. 34, 1848-1858 (2015)

44. Raiborg, C. \& Stenmark, H. The ESCRT machinery in endosomal sorting of ubiquitylated membrane proteins. Nature 458, 445-452 (2009).

45. Alpy, F. et al. STARD3 or STARD3NL and VAP form a novel molecular tether between late endosomes and the ER. J. Cell Sci. 126, 5500-5512 (2013).

46. Knoops, K. et al. SARS-coronavirus replication is supported by a reticulovesicular network of modified endoplasmic reticulum. PLoS Biol. 6, 1957-1974 (2008).

47. Memish, Z. A., Perlman, S., Van Kerkhove, M. D. \& Zumla, A. Middle East respiratory syndrome. Lancet 395, 1063-1077 (2020)

48. Chen, Q., Sun, L. \& Chen, Z. J. Regulation and function of the cGAS-STING pathway of cytosolic DNA sensing. Nat. Immunol. 17, 1142-1149 (2016).

49. He, Y., Li, B., Wang, C. \& Mao, S. X. Direct observation of dual-step twinning nucleation in hexagonal close-packed crystals. Nat. Commun. 11, 2483 (2020).

50. Motwani, M., Pesiridis, S. \& Fitzgerald, K. A. DNA sensing by the cGAS-STING pathway in health and disease. Nat. Rev. Genet. 20, 657-674 (2019).

51. Keren-Shaul, H. et al. A unique microglia type associated with restricting development of Alzheimer's disease. Cell 169, 1276-1290.e17 (2017).

52. Gao, Y. et al. Lipoprotein lipase maintains microglial innate immunity in obesity. Cell Rep. 20, 3034-3042 (2017).

53. Gong, H. et al. Lipoprotein lipase (LPL) is associated with neurite pathology and its levels are markedly reduced in the dentate gyrus of Alzheimer's disease brains. J. Histochem. Cytochem. 61, 857-868 (2013).

54. Strickland, D. K., Kounnas, M. Z. \& Argraves, W. S. LDL receptor-related protein: a multiligand receptor for lipoprotein and proteinase catabolism. FASEB J. 9, 890-898 (1995).

55. Marzolo, M. P., von Bernhardi, R., Bu, G. \& Inestrosa, N. C. Expression of a2macroglobulin receptor/low density lipoprotein receptor-related protein (LRP) in rat microglial cells. J. Neurosci. Res. 60, 401-411 (2000).

56. Yang, L. et al. LRP1 modulates the microglial immune response via regulation of JNK and NF-KB signaling pathways. J. Neuroinflammation 13, 1-13 (2016).

57. Brifault, C., Kwon, H., Campana, W. M. \& Gonias, S. L. LRP1 deficiency in microglia blocks neuro-inflammation in the spinal dorsal horn and neuropathic pain processing. Glia 67, 1210-1224 (2019).

58. Rauch, J. N. et al. LRP1 is a master regulator of tau uptake and spread. Nature 580, 381-385 (2020).

59. Konishi, H. \& Kiyama, H. Microglial TREM2/DAP12 signaling: a doubleedged sword in neural diseases. Front. Cell. Neurosci. 12, 1-14 (2018).

60. Deczkowska, A., Weiner, A. \& Amit, I. The physiology, pathology, and potential therapeutic applications of the TREM2 signaling pathway. Cell 181, 1207-1217 (2020).

61. Gratuze, M., Leyns, C. E. G. \& Holtzman, D. M. New insights into the role of TREM2 in Alzheimer's disease. Mol. Neurodegener. 13, 1-16 (2018).

62. Del-Aguila, J. L. et al. Assessment of the genetic architecture of Alzheimer's disease risk in rate of memory decline. J. Alzheimer's Dis. 62, 745-756 (2018).

63. Leyns, C. E. G. et al. TREM2 function impedes tau seeding in neuritic plaques. Nat. Neurosci. 22, 1217-1222 (2019).

64. Lee, S.-H. et al. Trem 2 restrains the enhancement of tau accumulation and neurodegeneration by $\beta$-amyloid pathology. Neuron 109, 1283-1301.e6 (2021).

65. Bemiller, S. M. et al. TREM2 deficiency exacerbates tau pathology through dysregulated kinase signaling in a mouse model of tauopathy. Mol. Neurodegener. 12, 1-12 (2017).

66. Deinhardt, K. Neuronal gatekeeper for dementia protein. Nature 580, 326-327 (2020).

67. Asai, H. et al. Depletion of microglia and inhibition of exosome synthesis halt tau propagation. Nat. Neurosci. 18, 1584-1593 (2015).

68. Hutton, M. et al. Association of missense and $5^{\prime}$-splice-site mutations in tau with the inherited dementia FTDP-17. Nature 393, 702-705 (1998).
69. Bugiani, O. et al. Frontotemporal dementia and corticobasal degeneration in a family with a P301S mutation in Tau. J. Neuropathol. Exp. Neurol. 58, 667-677 (1999)

70. Heppner, F. L., Ransohoff, R. M. \& Becher, B. Immune attack: the role of inflammation in Alzheimer disease. Nat. Rev. Neurosci. 16, 358-372 (2015).

71. Clark, I. A. \& Vissel, B. Amyloid $\beta$ : one of three danger-associated molecules that are secondary inducers of the proinflammatory cytokines that mediate Alzheimer's disease. Br. J. Pharmacol. 172, 3714-3727 (2015).

72. Venegas, C. \& Heneka, M. T. Danger-associated molecular patterns in Alzheimer's disease. J. Leukoc. Biol. 101, 87-98 (2017).

73. Saez-Atienzar, S. \& Masliah, E. Cellular senescence and Alzheimer disease: the egg and the chicken scenario. Nat. Rev. Neurosci. 21, 433-444 (2020).

74. Reinert, L. S. et al. Sensing of HSV-1 by the cGAS-STING pathway in microglia orchestrates antiviral defence in the CNS. Nat. Commun. 7, 13348 (2016).

75. Purice, M. D. \& Taylor, J. P. Linking hnRNP function to ALS and FTD pathology. Front. Neurosci. 12, 1-12 (2018).

76. Dormann, D. et al. ALS-associated fused in sarcoma (FUS) mutations disrupt transportin-mediated nuclear import. EMBO J. 29, 2841-2857 (2010).

77. Mudher, A. et al. What is the evidence that tau pathology spreads through prion-like propagation? Acta Neuropathol. Commun. 5, 99 (2017).

78. Mayor, S. \& Pagano, R. E. Pathways of clathrin-independent endocytosis. Nat. Rev. Mol. Cell Biol. 8, 603-612 (2007).

79. Brettschneider, J., Del Tredici, K., Lee, V. M. Y. \& Trojanowski, J. Q. Spreading of pathology in neurodegenerative diseases: a focus on human studies. Nat. Rev. Neurosci. 16, 109-120 (2015).

80. Guerreiro, R. et al. TREM2 variants in Alzheimer's disease. N. Engl. J. Med. 368, 117-127 (2013).

81. Jonsson, T. et al. Variant of TREM2 associated with the risk of Alzheimer's disease. N. Engl. J. Med. 368, 107-116 (2013).

82. Wang, Y. et al. TREM2 lipid sensing sustains the microglial response in an Alzheimer's disease model. Cell 160, 1061-1071 (2015).

83. Zhong, L. et al. Soluble TREM 2 induces inflammatory responses and enhances microglial survival. J. Exp. Med. 214, 597-607 (2017).

84. Umeda, T. et al. Rifampicin is a candidate preventive medicine against amyloid- $\beta$ and tau oligomers. Brain 139, 1568-1586 (2016).

85. Jiang, W. et al. Infiltration of CCR $2+$ Ly6C high proinflammatory monocytes and neutrophils into the central nervous system is modulated by nicotinic acetylcholine receptors in a model of multiple sclerosis. J. Immunol. 196, 2095-2108 (2016).

86. Brabazon, F., Bermudez, S., Shaughness, M., Khayrullina, G. \& Byrnes, K. R The effects of insulin on the inflammatory activity of BV2 microglia. PLoS ONE 13, 1-13 (2018).

\section{Acknowledgements}

We thank Huang Yong, Mao Takata, and Sumire Takayama for technical assistance. This work was supported by Grant-in-Aid for Scientific Research from Japan Society for Promotion of Science (JSPS) $(16 \mathrm{H} 02655 ; 19 \mathrm{H} 01042)$ and a Grant-in-Aid for Scientific Research on Innovative Areas (Foundation of Synapse and Neurocircuit Pathology, 22110001/22110002) from the Ministry of Education, Culture, Sports, Science and Technology of Japan (MEXT) to H.O.

\section{Author contributions}

M.J., H.S., H.T. and K.F.: data curation and writing-original draft and revised draft. H.H.: computational analysis and writing-original draft and revised draft. T.O., S.O. and K.N.: data curation. Y.Y. X.J., and K.K.: data curation. M.M.: resources, data curation and writing-original draft. H.O.: conceptualization, supervision, funding acquisition, project administration, and writing original draft and revised draft.

\section{Competing interests}

The authors declare no competing interests.

\section{Additional information}

Supplementary information The online version contains supplementary material available at https://doi.org/10.1038/s41467-021-26851-2.

Correspondence and requests for materials should be addressed to Hitoshi Okazawa.

Peer review information Nature Communications thanks Albert La Spada and the other, anonymous, reviewer(s) for their contribution to the peer review of this work. Peer reviewer reports are available.

Reprints and permission information is available at http://www.nature.com/reprints

Publisher's note Springer Nature remains neutral with regard to jurisdictional claims in published maps and institutional affiliations. 
(c) (i) Open Access This article is licensed under a Creative Commons Attribution 4.0 International License, which permits use, sharing, adaptation, distribution and reproduction in any medium or format, as long as you give appropriate credit to the original author(s) and the source, provide a link to the Creative Commons license, and indicate if changes were made. The images or other third party material in this article are included in the article's Creative Commons license, unless indicated otherwise in a credit line to the material. If material is not included in the article's Creative Commons license and your intended use is not permitted by statutory regulation or exceeds the permitted use, you will need to obtain permission directly from the copyright holder. To view a copy of this license, visit http://creativecommons.org/ licenses/by/4.0/.

(C) The Author(s) 2021 